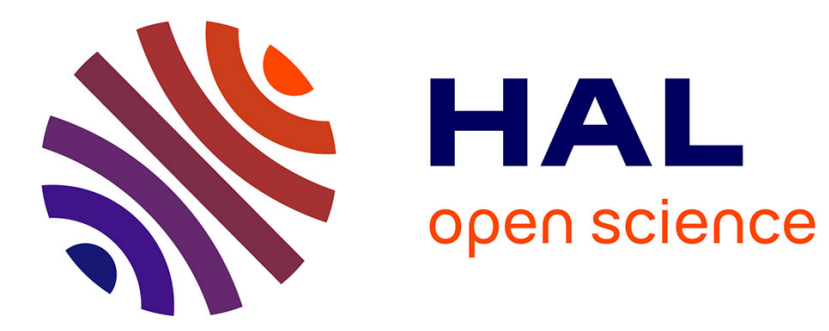

\title{
La méthode des choix multi-attributs appliquée aux Monts d'Arrée
}

Jeanne Dachary-Bernard

\section{To cite this version:}

Jeanne Dachary-Bernard. La méthode des choix multi-attributs appliquée aux Monts d'Arrée. Cahiers d'Economie et de Sociologie Rurales, 2007, 84-85, pp.133-166. hal-01201152

\section{HAL Id: hal-01201152 \\ https://hal.science/hal-01201152}

Submitted on 17 Sep 2015

HAL is a multi-disciplinary open access archive for the deposit and dissemination of scientific research documents, whether they are published or not. The documents may come from teaching and research institutions in France or abroad, or from public or private research centers.
L'archive ouverte pluridisciplinaire HAL, est destinée au dépôt et à la diffusion de documents scientifiques de niveau recherche, publiés ou non, émanant des établissements d'enseignement et de recherche français ou étrangers, des laboratoires publics ou privés. 


\section{La méthode des choix multi-attributs appliquée aux Monts d'Arrée}

Jeanne DACHARY-BERNARD 


\section{Jeanne DACHARY-BERNARD *}

\section{An application of the choice experiment method to the Monts d'Arrée}

Summary - Rural areas produce landscape amenities that improve users' welfare. Because people's demand for such amenities is growing, public decision makers have to deal with it. Knowing the benefits of landscape changes is useful for decision makers in order to belp them in their decision process.

Economic valuation of landscape changes is implemented in this article with the choice experiment method. This technique asks respondents what they prefer between different hypothetical landscape scenarios, each of them illustrating the landscape that would be if a particular political measure is taken. We apply this approach to the Monts d'Arrée landscape in Finistère where landscape changes are particularly interesting and decision makers have to take into account such landscape issues in their debate.

The economic model used gives the implicit prices of the landscape attributes of the study. From them, it calculates the consumer surplus derived from different landscape bypothetical programs since a landscape measure usually deals with more than one attribute.

Key-words: economic valuation, choice experiment metbod, landscape, preferences, public decision

\section{La méthode des choix multi-attributs appliquée aux Monts d'Arrée}

Résumé - Les espaces ruraux fournissent des aménités paysagères qui procurent du bien-être à leurs usagers. La demande sociale croissante en faveur de telles aménités justifie l'intervention des pouvoirs publics. Or, il est nécessaire à ce stade de connaître les bénéfices que provoqueraient des transformations paysagères.

L'évaluation économique du paysage que nous développons ici s'appuie sur la méthode des choix multiattributs. Celle-ci consiste à interroger les individus sur leurs préférences face à plusieurs hypothétiques scénarii paysagers, illustrant chacun une situation paysagère qui prévaudrait sous l'effet de mesures politiques particulières. Nous l'appliquons au paysage rural des Monts d'Arrée (Finistère) dont les problématiques paysagères sont réelles et vis-à-vis desquelles des mesures politiques sont envisagées.

Le modèle économique proposé produit les prix implicites des différents attributs paysagers considérés et permet d'en inférer les surplus attendus de différents programmes paysagers potentiels, une mesure paysagère ne concernant, en effet, rarement qu'un attribut mais plusieurs simultanément.

Mots-clés : évaluation ex ante, méthode des choix multi-attributs, paysage, préférences, aide à la décision

* CEMAGREF, Groupement de Bordeaux, 50 avenue de Verdun, 33612 Cestas-Gazinet e-mail : Jeanne.Dachary-Bernard@bordeaux.cemagref.fr 
$\mathrm{L}$ ES QUESTIONS environnementales sont aujourd'hui au cœur des préoccupations des populations et parmi elles, le paysage fait l'objet d'un intérêt particulier. Il est en effet considéré comme véhicule de la qualité de l'environnement mais également envisagé comme source d'agrément individuel et d'attractivité territoriale. Pour reprendre les termes employés dans la Convention européenne du Paysage (Conseil de l'Europe, 2000), le paysage est "un élément essentiel du bien-être individuel et social et de la qualité de vie des populations. Il participe de manière importante à l'intérêt général sur les plans culturel, écologique, environnemental et social et constitue une ressource favorable à l'activité économique, avec le tourisme notamment». Ce bien public connaît d'importantes transformations qui inquiètent, interpellent les populations et justifient une action publique de protection. Des textes de loi ont certes été adoptés, mais les acteurs politiques cherchent également à prendre en compte cette variable dans leurs décisions.

Bien que l'évaluation économique soit fortement critiquée pour ce genre de problématiques environnementales (Godard, 2004) et principalement pour des raisons de monétarisation des coûts et bénéfices non monétaires, nous considérons que l'évaluation économique est à ce stade un outil d'aide à la décision nécessaire, même s'il s'agit d'associer une valeur monétaire à des composantes qui ne sont pas « commensurables » (Aldred, 2006).

Dans une telle approche dite ex ante, l'évaluation permet de fournir aux décideurs des indicateurs monétaires des coûts et bénéfices attendus de certaines mesures politiques. L'évaluation consiste alors à traduire, sous forme monétaire, les valeurs associées au bien considéré. On distingue à ce titre les valeurs d'usage des valeurs de non-usage ${ }^{1}$ : les premières font référence aux fonctions esthétiques, récréatives et de cadre de vie du bien, les secondes sont rattachées à des considérations morales (valeur d'existence, d'héritage). Pour illustrer cette distinction fondamentale, citons le cas d'un produit alimentaire : la valeur d'usage sera liée à la consommation que l'on a de ce bien (qualité du produit, goût, packaging adapté à l'usage que l'on souhaite en avoir...); la valeur de non-usage fera référence aux schémas mentaux que ce produit nous évoque (mode de production « commerce équitable », " bio »...). Cet exemple ne reflète que partiellement le cas qui nous occupe ici puisque, à la différence du bien alimentaire, le paysage ne s'achète pas sur un marché. Sa valeur existe, mais elle n'est pas monétaire. L'emploi de méthode d'évaluation est alors indispensable pour lui associer une valeur monétaire, nécessaire au processus de choix des décideurs. Parmi les méthodes d'évaluation économique disponibles, les méthodes directes, qui interrogent directement les agents sur leurs préférences, permettent de s'attacher aux deux types de valeurs (même si elles peinent à clairement distinguer la part d'usage de la part de non-usage des valeurs ainsi révélées). Les méthodes indirectes ne peuvent approcher que les valeurs d'usage du bien étudié. Là où les premières techniques consistent à créer un marché hypothétique vis-à-vis duquel l'enquêté doit pouvoir se positionner pour répondre aux questions, les secondes méthodes utilisent

\footnotetext{
${ }^{1}$ Cette distinction se trouve également dans la littérature sous la forme «valeur d'usage actif/ d'usage passif ». Nous emploierons les deux indifféremment.
} 
le marché existant d'un bien qui est choisi parce que complémentaire du bien (non marchand) qui mobilise l'évaluation. S'agissant du paysage, il apparait nécessaire de pouvoir s'intéresser simultanément aux valeurs d'usage et de non-usage puisque ces dernières sont supposées relativement importantes au sein de la valeur économique totale du paysage (Graves, 1991). Nous privilégierons donc une méthode d'évaluation directe.

Mais le paysage n'est pas un objet d'étude standard en économie. Il convient donc de le positionner dans le champ de l'économie avant d'être en mesure de pouvoir choisir la méthode d'évaluation la plus adaptée. Cet effort de clarification quant à la nature économique du paysage a suivi une démarche qualifiée par Wilson en 1998 de «consilience» (Wilson, 1998). Ainsi, les hypothèses formulées quant à la caractérisation économique du paysage ont cherché à rester cohérentes et respectueuses du savoir des autres sciences sur ce même objet d'étude. La nature économique du paysage finalement retenue n'a pas la prétention d'être exhaustive, mais elle a pour vocation de définir l'objet d'étude par la discipline qui cherche à l'évaluer. Le paysage, produit d'une offre et satisfaisant une demande, est donc envisagé comme un bien économique. C'est plus particulièrement un bien économique non marchand, comme c'est le cas de nombreux biens environnementaux. Par ailleurs, il se compose de plusieurs éléments naturels aux interactions variables. Les transformations subies par l'une de ses composantes provoque une transformation notable du paysage dans sa globalité. Cette caractéristique confere au paysage une dimension multi-dimensionnelle, ce qui se traduira en termes économiques par un qualificatif de bien multi-attributs.

Cette dernière dimension du paysage est le point d'ancrage de l'analyse. C'est pourquoi la théorie mobilisée à ce stade est la théorie du consommateur de Lancaster (1971): le paysage est source d'agrément ou d'utilité pour l'individu du fait des différents attributs qui le composent ${ }^{2}$.

Compte tenu de l'approche lancasterienne retenue pour caractériser la nature économique du paysage, la méthode d'évaluation employée doit également suivre la même démarche multi-attributs. C'est pour cette raison que notre choix s'est porté sur la méthode des choix multi-attributs (MCMA) ou «choice experiment method». Il s'agit, en effet, non seulement d'une technique directe judicieuse pour s'attacher aux valeurs d'usage et de non-usage associées au paysage, mais qui plus est d'une technique considérant les transformations du bien comme étant générées par les changements connus par un ou plusieurs des attributs du bien.

Nous reviendrons dans une première partie sur un tel choix méthodologique, ses implications en terme de modélisations retenues et les caractéristiques de sa mise en œuvre sur le paysage des Monts d'Arrée (Finistère).

La seconde partie présentera les résultats de l'estimation en termes de préférences paysagères, notamment au travers du calcul des prix implicites qui découlent

\footnotetext{
2 Des travaux précédents (Dachary-Bernard, 2005) ont apporté un regard théorique plus approfondi sur cette dimension multi-attributs du paysage.
} 
directement de l'estimation faite. La discussion qui suivra procèdera par comparaison des résultats de différents groupes d'usagers (touristes, résidents permanents et secondaires), approche comparative intéressante dans le cadre d'une aide à la décision publique.

Enfin, la dernière partie élargira les résultats afin de fournir des indicateurs de bien-être agrégés pouvant supporter les décisions publiques. Plusieurs programmes paysagers seront simulés pour permettre d'envisager à quel niveau et comment les indicateurs calculés interviennent dans le processus public de décision. Ces résultats donneront également lieu à une analyse comparative des différents groupes d'usagers enquêtés, ce qui situe directement ce travail dans la continuité des travaux antérieurs (Dachary-Bernard, 2005) qui se sont plus spécifiquement attachés à estimer la valeur économique du paysage pour le seul groupe des touristes.

\section{Choix méthodologique et modélisation retenue}

La MCMA a été développée et fortement utilisée dans le domaine de l'économie des transports et du marketing à partir des années 1980 (Louviere and Woodworth, 1983). Son usage en management environnemental est, quant à lui, plus récent puisqu'il a été initié par Adamowicz et al. (1994) dont l'étude traitait de l'évaluation des préférences récréatives pour deux rivières canadiennes de l'Alberta. D'autres travaux ont suivi, notamment celui de Boxall et al. (1996) qui s'est intéressé à la chasse récréative (à l'élan) dans l'Alberta. Cependant, jusqu'à la fin du siècle dernier, seules les valeurs d'usage actif des biens sont prises en compte. La première étude à retenir et étudier des valeurs d'usage passif est celle menée par Adamowicz et al. (1998) qui traite de la protection de forêts dans ce même état. Morrisson et al. (1998) se sont, quant à eux, intéressés à la gestion des zones humides et Hanley et al., aux zones protégées du Royaume-Uni (ESA : «Environmentally Sensitive Areas ») estimant les préférences pour les paysages de forêts (1998a, 1998b).

Ces travaux ont permis de révéler cette méthode d'évaluation et de nombreux ouvrages ont suivi, décrivant plus en détails les aspects théoriques et techniques associés à la MCMA (Bennett and Blamey, 2001 ; Bateman et al., 2002).

\section{La méthode des choix multi-attributs}

Selon la théorie du consommateur de Lancaster (1971), la satisfaction que retire un individu de la consommation d'un bien s'explique notamment par les différents éléments constitutifs de ce bien. Cette approche multi-attributs est au cœur de la démarche méthodologique retenue puisque la MCMA consiste, dans un premier temps, à décomposer le bien à évaluer en plusieurs attributs principaux et à déterminer pour chacun d'eux les niveaux (états) qu'ils sont susceptibles de prendre. Un autre attribut est ajouté : il s'agit d'un attribut monétaire pour lequel plusieurs niveaux sont également définis. Cet attribut permet de prendre en compte la contrainte budgétaire du consommateur dans la démarche de choix et présente l'avantage, par rapport à d'autres techniques d'évaluation directes, de ne pas attendre de l'individu une construction de la valeur qu'il associe au bien. 
La seconde étape du protocole de mise en ouvre de la MCMA concerne la construction des expériences que l'on présente aux enquêtés. Pour ce faire, on adopte un processus de génération fractionnel des expériences (Kuhfeld, 2000) qui consiste, en respectant des critères statistiques bien définis (dont le critère d'orthogonalité), à combiner les différents attributs (et leurs différents niveaux) pour générer plusieurs scénarii, chaque scénario traduisant un état spécifique du bien étudié (au regard de chacun de ses attributs et de l'attribut monétaire) qui prévaudrait sous l'effet d'une mesure publique particulière. A ce stade, il est important de noter que l'attribut monétaire est considéré au même titre que les autres attributs du bien pour générer les scénarii, ce qui implique que les scénarii qui résultent de ce processus de génération peuvent très bien illustrer des mesures politiques coûteuses, mais pour lesquelles l'attribut monétaire associé est à son niveau le plus bas. Cette indépendance entre les attributs est fondamentale dans la construction des expériences, et permet justement de tester le rôle de contrainte budgétaire des agents lors de l'enquête. Ces scénarii, ou alternatives, sont ensuite regroupés au sein d'ensembles. Ainsi, chaque expérience présentée aux enquêtés correspond généralement à un ensemble de trois scénarii. Chacun des deux premiers scénarii représente la situation (hypothétique) qui prévaudrait suite à la mise en place d'une mesure politique particulière. Ces scénarii associent donc à chaque attribut considéré un certain niveau (ou état). Le troisième scénario de l'ensemble, qualifié de statu quo, illustre la situation de non-intervention publique. L'agent devra choisir le scénario qu'il préfere parmi les trois proposés, et cette expérience de choix se renouvelle plusieurs fois consécutives face à des scénarii alternatifs différents. Grâce à ce protocole méthodologique itératif, l'évaluateur dispose, en fin d'exercice, d'un éventail de préférences révélées, ce qui laisse supposer que la préférence réelle de l'individu pour le bien évalué est mieux approchée que s'il avait été demandé à l'enquêté de ne faire qu'un seul choix.

\section{Application au paysage des Monts d'Arrée}

Ce choix méthodologique a trouvé comme support empirique le paysage des Monts d'Arrée dans le Finistère ${ }^{3}$. Le terrain d'étude est une zone du Parc naturel régional d'Armorique (PNRA) qui s'étend sur 43000 ha, soit la surface de 14 communes rurales. Une certaine dualité caractérise le paysage de cette zone: au sud de la crête des Monts se trouve l'espace symbolique et sauvage des Monts d'Arrée composé de landes tourbeuses (le marais du Yeun Elez); au nord des Monts, un espace différent dominé par l'activité agricole qui structure le paysage en prairies et terres cultivables parcellées par des haies bocagères et talus caractéristiques du paysage emblématique de la Bretagne centrale (Gourmelen, 2002).

Cet espace connaît plusieurs enjeux relatifs aux transformations paysagères. La partie nord a connu il y a plusieurs années un remembrement qui a engendré

3 Ce travail a bénéficié, dans le cadre du Projet de recherche S3E - Sciences économiques et environnement - (2002), du financement du ministère de l'Ecologie et du Développement durable. 
arrachages de haies et de talus. Cette transformation du paysage bocager a eu pour effet, à la fois, la perte d'un cadre de vie, une dégradation paysagère, mais également l'érosion des sols, l'appauvrissement des milieux naturels et la pollution de l'eau. Même si le bocage breton est sans doute l'un des mieux conservés en France, la préservation, voire la reconstruction, d'une trame bocagère est aujourd'hui en question au niveau des instances de décision locales (Parcs naturels régionaux de France, 2000). De son côté, la zone sud connaît un double phénomène de boisement : la lande sèche s'enrésine sous l'effet d'initiatives individuelles et la lande humide voit se développer les friches avec l'abandon des terres. Cette seconde problématique de boisement spontané est affichée comme un enjeu pour le PNRA qui souhaite que ces landes gardent leur caractère traditionnel et qui incite pour cela à l'entretien des terres.

Face à de tels enjeux paysagers, reconnus par les pouvoirs publics, on peut se demander quels effets en termes de bien-être auraient des mesures paysagères destinées à sauvegarder le paysage.

Nous avons donc cherché à estimer la valeur économique que les individus accordent au paysage des Monts d'Arrée. Précisons que si l'emploi de la méthode des choix multi-attributs permet, comme nous l'avons précédemment exposé, de prendre en compte les valeurs d'usage actif et d'usage passif associées à ce bien, les individus concernés par cette enquête sont des « usagers » en ce sens qu'ils sont effectivement au contact du paysage étudié. Ils peuvent de ce fait valoriser le paysage à partir de l'usage qu'ils en font (valeurs d'usage in situ) ou du non-usage qu'ils en ont (valeurs de non-usage ou d'usage passif). En revanche, les «non-usagers », définis comme étant des individus n'ayant jamais été au contact de ce paysage breton, n'ont pas été considérés. Non que ce paysage n'ait aucune valeur pour eux, puisqu'ils peuvent en effet lui associer des valeurs de non-usage, mais l'évaluation n'a pas pris en compte cette source potentielle de valeur.

Nous nous sommes donc intéressés aux préférences paysagères, d'une part, de touristes, enquêtés de façon aléatoire au cours de l'été 2002, et, d'autre part, de la population de résidents (permanents et secondaires) rencontrés pendant le printemps 2003 après échantillonnage. Les données traitées portent au final sur 262 touristes et 345 résidents (dont $17 \%$ non permanents).

Outre des questions comportementales et socio-économiques, le cœur du questionnaire s'est attaché à présenter les différents scénarios paysagers possibles pour les Monts d'Arrée. Les différents attributs paysagers retenus pour construire de telles alternatives de choix ont été déterminés sur la base d'avis d'experts, ainsi qu'à partir des travaux réalisés deux ans plus tôt sur ce paysage (Le Floch, 2000). Les différents niveaux qui peuvent être pris par chaque attribut ont été définis à partir de considérations écologiques, de façon à ce que les scénarii constitués traduisent des situations paysagères réalistes. Chaque attribut peut prendre trois états distincts, le dernier niveau étant supposé « le meilleur » au regard du paysage traditionnel de la zone. Le tableau 1 ci-dessous récapitule les niveaux pris par chacun de ces trois attributs.

Comme le nécessite la démarche d'évaluation multi-attributs retenue, un attribut monétaire a été ajouté. Il s'agit, concernant la population de touristes, d'un 
supplément de taxe de séjour et pour les résidents, d'une augmentation de taxe d'habitation. Les trois niveaux que peuvent prendre ces attributs ont été fixés sur la base du montant, déterminé localement, des taxes ci-dessus (les données de référence se situent à l'échelle de la commune pour la taxe de séjour et à l'échelle départementale et régionale pour la taxe d'habitation). Ils sont également présentés dans le tableau 1 ci-dessous.

Seuls les attributs monétaires, faisant référence à des variations de taxes, sont d'ordre quantitatif. En effet, les attributs paysagers sont relatifs à des éléments esthétiques du paysage et les niveaux qu'ils peuvent prendre sont donc des grandeurs qualitatives (selon l'effet visuel produit). C'est pour faciliter la compréhension de l'exercice par les enquêtés que les différents scénarii ont été illustrés par des photos accompagnées d'une brève légende. Un exemple d'expérience telle que proposée aux résidents est proposée en annexe A1. La question complète qui leur a été adressée au cours du questionnaire au moment d'annoncer les expériences de choix est reproduite en annexe $\mathrm{A} 0$.

Un processus de génération fractionnel a été implémenté sous logiciel SAS pour créer les différents scénarii paysagers et les regrouper au sein d'ensembles: six expériences de trois scénarii chacune ont été finalement soumises au choix des enquêtés. Le troisième scénario est commun à toutes les expériences proposées : il s'agit du scénario de référence appelé «statu quo» dans lequel les attributs paysagers prennent le niveau qui prévaut lorsque aucune intervention n'est réalisée sur

Tableau 1. Liste des attributs et niveaux d'attributs retenus

\begin{tabular}{ll}
\hline \multicolumn{1}{c}{ Attributs } & \multicolumn{1}{c}{ Niveaux } \\
\hline Lande & très arborée \\
& peu arborée \\
& rase \\
\hline Bocage & non bocager \\
& moyennement bocager \\
& très bocager \\
\hline Bâtiments agricoles & non intégrés \\
& peu intégrés \\
\hline Prix (attribut monétaire) : & bien intégrés \\
Touristes : sup. de taxe de séjour & \\
& $0,10 €$ \\
Résidents : sup. de taxe d'habitation & $0,20 €$ \\
& $0,30 €$ \\
& $15 €$ \\
\hline
\end{tabular}


l'attribut ${ }^{4}$, c'est-à-dire les niveaux 1 , et l'attribut monétaire prend une valeur nulle puisque aucune action paysagère n'est à financer.

\section{Modélisation économique retenue}

Comment l'enquêté fait-il ses choix de préférences? Contrairement à une approche de maximisation de l'utilité sous contrainte budgétaire, l'approche économique sous-jacente est celle des modèles à choix discrets et de la théorie de l'utilité aléatoire (Thurstone, 1927 ; Mcfadden, 1974). L'hypothèse de base consiste à poser que, confronté à plusieurs choix ou alternatives, l'agent-consommateur va choisir l'option qui maximise son utilité sachant que cette utilité est en partie aléatoire. Ce caractère aléatoire ne traduit pas un manque de rationalité du consommateur dans son comportement, mais il reflète plutôt un manque d'information de l'observateur (l'évaluateur) quant aux caractéristiques des alternatives et/ou des consommateurs qui contribuent à expliquer le choix. On représente mathématiquement la fonction d'utilité de l'individu $n$ sous la forme suivante :

$$
\begin{array}{r}
U_{\text {in }}=V_{\text {in }}+\varepsilon_{\text {in }} \\
\text { avec } V_{\text {in }}=A_{i}+\beta^{\prime} x_{\text {in }}
\end{array}
$$

$U_{i}$ représente l'utilité de l'individu pour l'alternative $i, V_{i}$ est la composante systématique de l'utilité dite utilité «indirecte », et $\varepsilon_{i}$ est la composante aléatoire de l'utilité qui traduit les goûts particuliers non observés des individus. L'utilité indirecte est quant à elle considérée comme étant expliquée par des variables $x_{i}$ ainsi que par une constante spécifique à l'alternative $i$ notée $A_{i}$.

Partant de l'expression synthétique du modèle économique donnée en (1), l'individu $n$ choisira l'alternative $i$ d'un ensemble de choix $C$ composé de plusieurs autres alternatives $j$ si et seulement si :

$$
\begin{array}{r}
\quad U_{i n}>U_{j n}, \forall j \neq i \in C \\
\Leftrightarrow\left(V_{i n}+\varepsilon_{i n}\right)>\left(V_{j n}+\varepsilon_{j n}\right) \\
\Leftrightarrow\left(V_{i n}-V_{j n}\right)>\left(\varepsilon_{j n}-\varepsilon_{i n}\right)
\end{array}
$$

Ne pouvant observer le membre droit de cette inégalité, on s'intéresse à la probabilité d'occurrence d'une telle relation. Ainsi, la probabilité pour l'individu de choisir l'alternative $i$ parmi toutes celles $(j)$ de l'ensemble $C$ est donnée par :

\footnotetext{
${ }^{4}$ Ce qui ne correspond pas nécessairement à la situation actuelle des attributs.
} 


$$
\begin{aligned}
& P_{n}(i / C)=P_{n}\left[\left(V_{i n}-V_{j n}\right)>\left(\varepsilon_{j n}-\varepsilon_{i n}\right)\right], \forall i \neq j \in C \\
& \Leftrightarrow P_{n}(i / C)=P_{n}\left[\varepsilon_{j n}<\varepsilon_{i n}+\left(V_{i n}-V_{j n}\right)\right], \forall i \neq j \in C
\end{aligned}
$$

Estimée par la méthode du maximum de vraisemblance, cette probabilité sera intégrée dans la fonction de vraisemblance définie comme suit (avec $n$, mis pour l'individu) :

$$
\text { Ln } L=\sum_{n=1}^{N} \sum_{i=1}^{I} d_{n i} \log P_{n i}
$$

Cette expression (4) correspond à proprement parler au modèle d'utilité aléatoire (Louviere et al., 2000). Il reste néanmoins à convenir d'une distribution des éléments qui influencent cette relation de choix à l'utilité pour pouvoir estimer la fonction d'utilité. Ce choix de distribution va conditionner le modèle économétrique que nous retiendrons.

\section{Modélisation économétrique retenue}

A ce stade, plusieurs types de modèles à choix discrets peuvent être envisagés selon le type d'hypothèses formulées au regard du terme aléatoire (Train, 2002). Le fait de retenir une approche multi-attributs implique, par construction, d'utiliser le Logit. En effet, cet outil économétrique a été développé par McFadden afin d'apporter une formalisation à la théorie de l'utilité aléatoire. La recherche d'une certaine cohérence dans la démarche suivie nous incite donc à retenir un modèle Logit.

En revanche, plusieurs formes de Logit peuvent être envisagées (Greene, 2003). Nous retiendrons le modèle Logit multinomial qui prend en compte à la fois les variables caractéristiques de l'agent enquêté et les attributs du paysage étudié.

Nous supposerons, comme cela a souvent été fait dans la littérature, que les termes aléatoires sont indépendants et identiquement distribués (iid) selon la distribution d'extrême valeur de type I (EV1). Nous chercherons en conséquence à estimer la probabilité suivante :

$$
P_{i n}=\frac{\exp \left(V_{i n}\right)}{\sum_{j=1}^{J} \exp \left(V_{j n}\right)}=\frac{\exp \left(\beta^{\prime} x_{i}\right)}{\sum_{j=1}^{J} \exp \left(\beta^{\prime} x_{j}\right)}
$$

Précisons qu'il s'agit d'une hypothèse forte posée sur les termes aléatoires puisqu'ils sont supposés suivre tous la même distribution. On peut lever cette hypothèse en développant un Random Parameters Logit (ou «mixed logit»), dans lequel les composantes stochastiques peuvent suivre tout type de distribution 
statistique et permettre ainsi de prendre en compte l'hétérogénéité des enquêtés (Rigby and Burton, 2004). A l'instar d'autres travaux récents (Horne et al., 2005 ; Colombo et al., 2006), nous avons fait le choix de poursuivre sur un Logit standard, mais l'analyse pourrait être encore plus approfondie en développant un «mixed Logit ». Notons néanmoins à ce stade que de récents travaux ont comparé les pouvoirs prévisionnels de plusieurs modèles d'utilité aléatoire dont un «mixed Logit» et un Logit standard (Provencher and Bishop, 2004). Les auteurs concluent à la nonsupériorité des uns par rapport aux autres et s'étonnent de la meilleure qualité de prédiction du Logit standard dans certaines conditions. Ces conclusions confortent notre choix empirique.

Compte tenu des résultats de plusieurs études sur l'intérêt de la prise en compte des caractéristiques individuelles (des enquêtés) en sus des attributs du bien à évaluer (Hanley et al., 2000; Alvarez-Farizo and Hanley, 2002 ; Bergmann et al., 2006 ; Colombo et al., 2006), nous avons choisi de considérer dans notre modèle à la fois les attributs et quelques caractéristiques des enquêtés.

Pour chaque individu $n$, la fonction d'utilité indirecte associée au choix de l'alternative $i$ est donc de la forme :

$$
V_{i n}=A_{i}+\sum_{k} \beta_{i k} x_{i k}+\sum_{b} \gamma_{i b n}\left(z_{b n} * A_{i}\right)
$$

où $A_{i}$ est la constante spécifique à l'alternative $i$ (ASC), $\beta_{k}$ les paramètres à estimer pour les attributs $k(k=1, \ldots K)$ propres à l'alternative $i$ représentés par les variables $x_{i k}, \gamma_{i b n}$ les paramètres à estimer lors du choix de l'alternative $i$ pour les caractéristiques $h(b=1, \ldots H)$ propres à l'individu $n$ représentées par les variables $z_{b n}$ et intégrées dans le modèle en interaction avec les ASC.

Compte tenu de cette forme fonctionnelle (7), la probabilité (6) peut s'écrire de la façon suivante :

$$
P_{i n}=\frac{\exp \left(\beta^{\prime} x_{i}+\gamma_{i n}{ }^{\prime} z_{n}\right)}{\sum_{j=1}^{J} \exp \left(\beta^{\prime} x_{j}+\gamma_{j n}{ }^{\prime} z_{n}\right)}
$$

Concernant les attributs du modèle $\left(x_{i k}\right)$, ils ont été retenus compte tenu des considérations empiriques du paysage étudié. Ils ont été présentés plus haut, dans le tableau 1. Une précision doit être apportée à ce stade, concernant la manière d'intégrer les variables-attributs dans le modèle. Les trois attributs paysagers sont en effet des variables qualitatives discrètes dont les niveaux possibles traduisent des transformations multi-attributs notables du paysage. C'est pourquoi la distinction entre les deux types de transformations envisagées (passage du niveau 1 au niveau 2 ou du niveau 2 au niveau 3) doit pouvoir être prise en compte. Pour ce faire, le système de codage des variables employé, appelé «effect coding ", permet de régresser le niveau de l'utilité sur des niveaux d'attributs et non plus sur l'attribut (McVittie et al., 2001). Afin de capturer ces niveaux, pour chaque attribut à trois niveaux sont 
créées deux nouvelles variables aux effets codés $\left(\mathrm{VEC}_{2}\right.$ et $\left.\mathrm{VEC}_{3}\right)$ : $\mathrm{VEC}_{2}$ prendra la valeur 1 si le niveau 2 est pris par l'attribut en question et la valeur 0 sinon; $\mathrm{VEC}_{3}$ prendra la valeur 1 si l'attribut est à son niveau 3 et prendra une valeur nulle sinon ; enfin si l'attribut prend le niveau 1, considéré comme le niveau de référence «le moins bon ", $\mathrm{VEC}_{2}$ et $\mathrm{VEC}_{3}$ prendront chacune la valeur - 1. Il s'agit d'un système alternatif au codage employant des variables "dummies", dans lequel le niveau de référence est assigné de la valeur -1 et non de la valeur nulle comme dans le cas de dummy (Bech and Gyrd-Hansen, 2005). Autrement dit, dans le cas de cette étude, le choix du statu quo de la part du répondant est représenté par la valeur -1 prise simultanément pas les deux VEC de chaque attribut paysager. Ce système de codage ne s'applique bien évidemment pas à la variable prix qui est une variable numérique.

Pour les caractéristiques du modèle à prendre en compte $\left(z_{b n}\right)$, nous avons retenu sur des considérations d'ordre microéconomique les variables socio-économiques standards (sexe, âge, revenu et nombre d'enfants) (Carlsson et al., 2003) ainsi que des variables comportementales, pertinentes au regard du type d'individus enquêtés, telles que les nuitées pour les touristes, ou le type d'habitation (principale ou secondaire) pour les résidents. L'ensemble des variables individuelles initialement envisagées pour les modèles des touristes et des résidents est décrit en annexe A2. Cependant, après plusieurs développements économétriques, force a été de constater que certaines de ces caractéristiques individuelles devaient être enlevées du modèle initial car non significatives.

Ainsi, le modèle économétrique que nous avons estimé peut être formulé de la façon suivante sur le terrain d'étude des Monts d'Arrée :

$$
\begin{aligned}
V_{\text {in }}= & A_{i}+\beta_{2} l_{2}+\beta_{3} l_{3}+\delta_{2} b_{2}+\delta_{3} b_{3}+\eta_{2} b_{a 2} \\
& +\eta_{3} b_{a 3}+\pi p+\sum_{b} \gamma_{i b n}\left(z_{b n} * A_{i}\right)
\end{aligned}
$$

Les variables $l_{2}$ et $l_{3}, b_{2}$ et $b_{3}$, puis $b_{a 2}$ et $b_{a 3}$ sont les variables dichotomiques associées aux niveaux 2 et 3 de, respectivement, la lande, le bocage et le bâti ; la variable $p$ correspond à l'attribut monétaire.

Cependant, aucun a priori n'impose que le modèle économique intègre le prix sous forme linéaire simple (Hanley et al., 1998a) et nous avons finalement décidé, après avoir estimé l'équation (9), d'intégrer le prix sous forme quadratique ${ }^{5}$, aussi bien dans le modèle des touristes que dans celui des résidents. Ainsi, de manière générique, le modèle des préférences paysagères que nous allons chercher à estimer se formalise de la façon suivante (pour l'agent $n$ ):

5 Un LR test (ou «Log-Likelibood ratio test ») a été pratiqué et a permis de conclure aussi bien pour les résidents que pour les touristes que le modèle sous forme quadratique était préférable. Par souci de lisibilité, ni le modèle initial ni le résultat de ce test ne sont présentés ici. 


$$
\begin{aligned}
V_{\text {in }}= & A_{i}+\beta_{2} l_{2}+\beta_{3} l_{3}+\delta_{2} b_{2}+\delta_{3} b_{3}+\eta_{2} b_{a 2} \\
& +\eta_{3} b_{a 3}+\pi_{1} p+\pi_{2} p^{2}+\sum_{b} \gamma_{i b n}\left(z_{b n} * A_{i}\right)
\end{aligned}
$$

\section{Résultats et calculs des prix implicites}

Deux modèles distincts ont été estimés à partir des logiciels Limdep et Stata: l'un pour les touristes (262 individus), l'autre pour les résidents permanents et secondaires (345 au total).

\section{Estimation des modèles}

L'analyse des préférences paysagères des touristes ayant fait l'objet d'une publication antérieure (Dachary-Bernard, 2005), l'ensemble des résultats relatifs à cette catégorie d'usagers est présenté de manière synthétique en annexe $\mathrm{A} 3$ (même si quelques détails supplémentaires ont été ajoutés) et les résultats de l'estimation pour la population de résidents sont présentés dans le tableau 2 ci-après. Ces résultats seront analysés comparativement à ceux des touristes.

Dans les deux cas, les constantes (ASC) ont été estimées, mais exclues du modèle car non significatives. Ces ASC sont spécifiques aux alternatives autres que le statu $q u$, et traduisent le rôle des sources non observées de l'utilité propre à chaque

Tableau 2. Résultats des estimations du Logit multinomial quadratique aux effets codés pour

\begin{tabular}{|c|c|c|}
\hline \multicolumn{2}{|c|}{ Variables } & \multirow[t]{2}{*}{ Paramètres estimés } \\
\hline Attributs: & & \\
\hline Lande $_{2}$ & $\left(\beta_{2}\right)$ & $+0,59 * * *$ \\
\hline Lande $_{3}$ & $\left(\beta_{3}\right)$ & $-0,34 * * *$ \\
\hline Bocage $_{2}$ & $\left(\delta_{2}\right)$ & $-0,16^{*}$ \\
\hline Bocage $_{3}$ & $\left(\delta_{3}\right)$ & $+0,83 * * *$ \\
\hline Bâti-agricole 2 & $\left(\eta_{2}\right)$ & $-0,48 * * *$ \\
\hline Bâti-agricole $_{3}$ & $\left(\eta_{3}\right)$ & $+0,2 /$ \\
\hline Prix & $\left(\pi_{1}\right)$ & $+0,07 * * *$ \\
\hline $\operatorname{Prix}^{2}$ & $\left(\pi_{2}\right)$ & $-0,0009 * *$ \\
\hline \multicolumn{3}{|l|}{ Caractéristiques: } \\
\hline Revenu(ASC ${ }_{1}$ ) & $\left(\gamma_{41}\right)$ & $+0,29 * * *$ \\
\hline Revenu(ASC 2 ) & $\left(\gamma_{42}\right)$ & $+0,32 * * *$ \\
\hline RL2( ASC $\left._{1}\right)$ & $\left(\gamma_{51}\right)$ & $+0,17 /$ \\
\hline $\mathrm{RL} 2\left(\mathrm{ASC}_{2}\right)$ & $\left(\gamma_{52}\right)$ & $-0,37 *$ \\
\hline $\operatorname{RL} 3\left(\mathrm{ASC}_{1}\right)$ & $\left(\gamma_{61}\right)$ & $-0,54 * * *$ \\
\hline $\mathrm{RL} 3\left(\mathrm{ASC}_{2}\right)$ & $\left(\gamma_{62}\right)$ & $+0,24 /$ \\
\hline
\end{tabular}
les résidents

Significativité à $1 \%(* * *), 5 \%(* *), 10 \%(*)$ ou non-significativité $(/)$ 
alternative (Hensher et al., 2005). Elles représentent donc, toute chose égale par ailleurs, l'utilité de choisir une autre option que le statu quo: soit l'alternative 1 (scénario 1), soit l'alternative 2 (scénario 2). Notons que le statu quo traduit ici la non-intervention des pouvoirs publics, mais ce n'est pas la situation actuelle puisque certaines mesures paysagères sont d'ores et déjà prises sur la zone étudiée. Si de telles constantes sont non significatives, c'est que le choix d'une alternative autre que le statu quo ne génère pas significativement plus ou moins d'utilité que le choix du statu quo. Généralement, le statu quo reflétant la situation actuelle et les agents ayant une préférence pour le présent, un biais, qualifié de «statu quo bias », apparaît lorsque les enquêtés sont dans une situation de choix qui les incite à s'éloigner de leur situation actuelle (Louviere et al., 2000) : ils ont tendance à s'orienter spontanément vers une situation qui n'implique aucun changement pour eux, autrement dit le statu quo. Ce n'est semble-t-il pas le cas ici. Aussi, la technique adoptée par certains (Bonnieux et al., 2006) pour prendre en compte la signification particulière du statu quo dans l'esprit des gens n'a pas été jugée pertinente dans notre cas.

Les caractéristiques individuelles ont été incluses dans le modèle en interaction avec les ASC, d'où l'estimation de deux paramètres pour chaque variable selon que le scénario correspondant ait été présenté en première ou seconde position (parmi les deux scénarii alternatifs qui font face au statu quo).

Globalement, les signes des estimateurs sont conformes aux attentes.

$\mathrm{Au}$ regard de ces caractéristiques individuelles, notons que le revenu s'est avéré être la seule variable socioéconomique significative dans le modèle des résidents, et qu'elle est significative uniquement (et positivement) chez les résidents. Toute chose égale par ailleurs, les ménages-résidents les plus aisés retirent une satisfaction plus importante du choix d'un scénario que les autres résidents, ce qui va dans le sens de la théorie économique: l'effort d'une participation financière (quelle que soit la raison de cette participation) est moins lourd pour un individu fortuné. En revanche, le revenu joue moins pour les touristes, ce qui peut se comprendre: l'effort d'une participation financière n'a pas le même sens pour un touriste qui, de par sa situation de touriste, est dans une logique d'acceptation de la dépense.

La variable indiquant le type de résidence, que l'on supposait être importante, n'est pas significative dans la formulation initiale, d'où son exclusion du modèle. Cependant, le fait d'être en résidence principale ou secondaire pouvant avoir un impact sur l'utilité des résidents au niveau de leur préférence vis-à-vis de la lande ${ }^{6}$, nous avons intégré ce phénomène au modèle en croisant la variable «type de résidence » avec l'attribut "lande » de niveau 2 et de niveau 3. Ces deux variables, notées RL2 et RL3, définies comme étant le produit d'une variable socioéconomique et d'une variable-attribut, sont donc elles-mêmes des variables socioéconomiques intégrées au modèle par interaction avec les constantes (ASC). Elles sont significativement non nulles dans l'un des deux ordres présentés, ce qui justifie de

\footnotetext{
${ }^{6}$ Cette hypothèse a découlé du choix des scénarii paysagers faits par les enquêtés, mais qui ne sont pas reportés ici. Pour plus de détails, se référer à Dachary-Bernard, 2004.
} 
distinguer les préférences des résidents secondaires de celles des autochtones au regard de l'attribut lande.

La variable concernant le nombre d'enfants, non significative chez les résidents, est positivement significative chez les touristes. Elle souligne bien le fait que les arbitrages ne sont pas perçus par les touristes comme des efforts financiers (le prix correspondant à une personne séjournant, le coefficient de cette variable serait négatif), mais plutôt comme une logique de durabilité, de protection du paysage pour les générations futures.

Par ailleurs, en ce qui concerne les attributs du modèle, précisons que les niveaux 1 des attributs ne sont pas directement estimés dans le modèle, comme l'explique le principe de "l'effect coding» présenté plus haut. En revanche, il est possible de les déduire des estimations des niveaux 2 et 3 du même attribut: le coefficient associé au niveau 1 correspond à l'inverse de la somme des coefficients estimés pour les autres niveaux. Le tableau 3 ci-dessous présente les valeurs à prendre en compte, après calculs, pour les résidents :

- pour les niveaux 1 des attributs «bocage » et «bâti », le calcul a procédé par simple soustraction tel que proposé par l'effect coding,

- pour l'attribut de la lande, compte tenu de l'influence des variables RL2 et $\mathrm{RL} 3^{7}$ et de l'effect coding, les trois paramètres associés aux trois niveaux de la lande ont du être calculés.

Les résultats associés à la population de touristes, auxquels le lecteur pourra se référer en annexe A3 pour comprendre l'analyse comparative qui suit, distinguent également les paramètres estimés par le modèle de ceux qui ont été calculés.

Si les statistiques de student ont été calculées pour chacun de ces paramètres, seule leur éventuelle significativité est rapportée dans le tableau.

Les préférences paysagères des résidents s'orientent ainsi vers un espace agricole très bocager, pour lequel les niveaux 2 et 1 sont même négativement valorisés. Les touristes vont également dans ce sens, mais plus nuancés, ils acceptent la situation d'un bocage moyennement dense. En revanche, pour le bâti, les trois groupes

Tableau 3. Calculs des paramètres non estimés dans le modèle des résidents

\begin{tabular}{lccc}
\hline \multicolumn{1}{c}{ Variables } & Paramètres calculés & Résidents permanents & Résidents secondaires \\
\hline Lande $_{1}$ & $\beta_{1}$ & $+0,67 *$ & $-0,25 * * *$ \\
Lande $_{2}$ & $\beta_{2}$ & $+0,22 /$ & $+0,59 * * *$ \\
Lande $_{3}$ & $\beta_{3}$ & $-0,88^{* * *}$ & $-0,34 * *$ \\
Bocage $_{1}$ & $\delta_{1}$ & $-0,67 * * *$ & $-0,67 * * *$ \\
Bâti $_{1}$ & $\eta_{1}$ & $+0,28 /$ & $+0,28 /$ \\
\hline
\end{tabular}

Significativité à $1 \%(* * *), 5 \%(* *), 10 \%(*)$ ou non-significativité $(/)$

${ }^{7}$ La significativité de ces variables RL2 et RL3 implique la prise en compte dans les calculs des paramètres qui leurs sont associés, ce qui justifie la nouvelle notation des paramètres « globaux » associés à la lande : $\beta_{1}, \beta_{2}$ et $\beta_{3}$. 
d'agents préferent la situation d'une intégration paysagère parfaite, même si cette fois les touristes n'acceptent même pas une intégration partielle. A ce stade, un résultat surprenant doit être souligné concernant les résidents : il s'agit du coefficient positif associé au niveau de base du bâti. La différence de préférences réside dans la nuance: là où les touristes valorisent positivement de façon significative une intégration parfaite des bâtis, les résidents valorisent négativement une intégration partielle (le coefficient de bâti ${ }_{3}$ est certes positif, mais il n'est pas significatif dans le modèle). Concernant plus particulièrement la lande, les préférences des touristes et des résidents secondaires vont dans le même sens et differrent de celles des autochtones. Là où les locaux révèlent une forte préférence pour l'état très arboré de la lande (le paramètre est non significatif chez les touristes), les habitants secondaires et les touristes ont une préférence pour l'état intermédiaire de la lande. Ce résultat est intéressant, car il souligne une convergence des préférences des non-permanents (touristes et résidents secondaires) par rapport à celles de ceux qui habitent toute l'année sur la zone : une « expérience » du paysage pourrait-elle être à l'origine de ces divergences? Le fait que la variable «visitepassée » soit significative chez les touristes va dans ce sens: le fait de n'être jamais venu joue négativement sur le niveau d'utilité, laissant entrevoir cette même logique de connaissance du paysage comme variable explicative des valeurs qui lui sont associées.

\section{Calcul des prix implicites des attributs du paysage}

Ces paramètres estimés traduisent, certes, les préférences paysagères des enquêtés, mais ils ne peuvent pas être utiles sous cette forme aux décideurs. Il faut donc construire des indicateurs monétaires qui permettront de souligner les importances relatives que les individus accordent au paysage. Il s'agit des prix implicites (ou consentements à payer $(\mathrm{CAP})$ marginaux ${ }^{8}$ ) qui correspondent à des taux marginaux de substitution entre le niveau de l'attribut considéré et l'attribut monétaire. On définit donc le prix implicite du niveau $i$ de l'attribut $k\left(x_{k i}\right)$ par la formule suivante :

$$
P I_{k i}=-\frac{\partial V / \partial x_{k i}}{\partial V / \partial p}=-\frac{\tau_{k i}}{\pi_{1}+2 \pi_{2} \bar{p}}
$$

Le numérateur correspond à l'utilité marginale du niveau $i$ de l'attribut $k$. Le dénominateur, du fait de la relation quadratique de l'utilité au prix, est une fonction du niveau de ce prix. Compte tenu des valeurs pivots, nous avons choisi de prendre les valeurs de $0,20 €$ et de $40 €$ pour respectivement les touristes et les résidents, ce qui donne d'après nos données :

$$
(\partial V / \partial p)_{\text {touristes }}=-0,34 \text { et }(\partial V / \partial p)_{\text {résidents }}=-0,002 \text {. }
$$

8 On emploie ce terme de «marginal » pour le consentement à payer afin de faire référence aux utilités marginales impliquées dans son calcul. Cependant, cet usage est abusif dans le sens où le CAPm est associé à des transformations discrètes et non continues de l'attribut $j$ correspondant. 
Pour faciliter l'analyse comparative des prix implicites des différentes catégories d'individus considérées, les prix moyens ont été exprimés en euros par ménage et par an, ce qui a nécessité un ajustement ${ }^{9}$ pour les prix implicites des touristes (exprimés jusque-là en euros par personne et par nuit). Les CAP marginaux ainsi obtenus pour les résidents sont présentés dans le tableau 4 ci-dessous, et peuvent être comparés à ceux des touristes (dernière colonne en annexe A3). Les intervalles de confiance sont calculés par «parametric bootstrapping » tel que proposé par Krinsky (1981): il s'agit de simuler la distribution asymptotique des coefficients en considérant plusieurs tirages aléatoires d'une loi normale définie pour les paramètres estimés et leur matrice de variance-covariance. Nous avons généré ainsi 1000 observations et 1000 CAP marginaux ont donc été calculés pour chaque niveau d'attribut (Poe et al., 1997 ; Foster and Mourato, 2003). L'intervalle de confiance est donné par les percentiles, la borne inférieure correspondant au premier percentile $(10 \%)$ et la borne supérieure au dernier percentile $(90 \%)$.

On constate que, dans l'ensemble, les prix implicites des touristes pour les attributs paysagers sont plus élevés en valeur absolue que ceux des autres groupes enquêtés. Or, aucun a priori n'était posé à ce sujet puisque parmi les différentes

Tableau 4. Prix implicites (PI) des niveaux d'attributs paysagers selon les groupes d'individus

\begin{tabular}{|c|c|c|c|}
\hline & \multirow{2}{*}{ Niveau d'attribut } & \multicolumn{2}{|c|}{$\begin{array}{c}\text { PI moyen ( } € / \text { ménage/an) } \\
\text { ( } 95 \% \text { intervalle de confiance) }\end{array}$} \\
\hline & & Résidents permanents & Résidents secondaires \\
\hline Lande $_{1}$ & & $\begin{array}{c}+31,6 \\
(-121,9 ; 192,8)\end{array}$ & $\begin{array}{c}-11,9 \\
(-59,9 ; 37,2)\end{array}$ \\
\hline Lande $_{2}$ & & $\begin{array}{c}+13,7 \\
(-21,9 ; 46,8)\end{array}$ & $\begin{array}{c}+32,4 \\
(-86,6 ; 139,2)\end{array}$ \\
\hline Lande $_{3}$ & & $\begin{array}{c}-47,8 \\
(-222,4 ; 137) \\
\end{array}$ & $\begin{array}{c}-18,7 \\
(-83,3 ; 50,8) \\
\end{array}$ \\
\hline Bocage $_{1}$ & & \multicolumn{2}{|c|}{$\begin{array}{c}-37,5 \\
(-176 ; 111,6)\end{array}$} \\
\hline Bocage $_{2}$ & & \multicolumn{2}{|c|}{$\begin{array}{c}-7,4 \\
(-41,2 ; 28,4) \\
\end{array}$} \\
\hline Bocage $_{3}$ & & \multicolumn{2}{|c|}{$\begin{array}{c}+45 \\
(-142,6 ; 208,7)\end{array}$} \\
\hline Bâti & & \multicolumn{2}{|c|}{$\begin{array}{c}+16,9 \\
(-30,1 ; 75,9)\end{array}$} \\
\hline Bâti 2 & & \multicolumn{2}{|c|}{$\begin{array}{c}-27 \\
(-108,2 ; 63,1)\end{array}$} \\
\hline Bâti 3 & & \multicolumn{2}{|c|}{$\begin{array}{c}+7,9 \\
(-47,5 ; 53,6) \\
\end{array}$} \\
\hline
\end{tabular}

9 Nous avons considéré, d'après les statistiques descriptives de la population de touristes, que le ménage se composait en moyenne de 3,5 personnes et que ce ménage passait en moyenne 7,5 nuits sur la zone d'étude. 
études qui se sont intéressées à ces mêmes groupes d'individus aucune tendance générale ne s'était révélée : là où certains n'ont pu conclure quant à la supériorité des bénéfices attendus de certains individus plutôt que d'autres (Willis and Garrod 1993), d'autres ont montré que le CAP moyen des résidents est plus élevé que celui des visiteurs (Bullock and Kay, 1997), et d'autres, enfin ont conclu l'inverse (Hanley et al., 1998b). On peut dès lors considérer dans ce cas d'étude que les touristes sont prêts à payer globalement plus que les résidents pour pouvoir bénéficier d'une transformation multi-attributs du paysage concerné, mais il s'agit bien d'une conclusion de cas particulier: et elle n'est pas généralisable. Elle est néanmoins cohérente avec le fait que, d'après nos données, la population de touristes est plus fortement représentée dans les tranches de revenus supérieures que celle des résidents.

Si on regarde en détail la situation par attribut paysager, on remarque que le niveau 3 de la lande est associé pour les trois groupes d'individus à des consentements à payer marginaux négatifs, autrement dit des consentements à recevoir (CAR). La situation a priori la meilleure de cet attribut est donc associée à une perte de bien-être individuel pour les individus. Ils dégagent donc des préférences pour la lande arborée, cependant les touristes et résidents secondaires dégagent également un CAP marginal négatif pour lande ${ }_{1}$, ce qui signifie qu'ils préferent l'état intermédiaire de la lande. Ce résultat est intéressant au regard des mesures paysagères prises aujourd'hui sur la zone étudiée et qui consistent à entretenir la lande pour la maintenir rase. Ceci nous amène alors à constater que les actions publiques relatives au paysage de lande des Monts d'Arrée vont dans le sens inverse des préférences des usagers.

Concernant le bocage, tous les groupes d'enquêtés révèlent, sans surprise, une préférence pour un espace agricole très bocager, et un CAR pour un espace agricole sans bocage. Le bâtiment agricole est également préféré dans son état «le meilleur » c'est-à-dire bien intégré, même si on peut s'interroger vis-à-vis du résultat associé aux résidents: le prix implicite du bâti non intégré est positif, ce qui est contradictoire avec le résultat également positif pour le niveau bien intégré de ce même attribut.

Si ces indicateurs des préférences paysagères sont déjà d'une utilité certaine pour les décideurs pour se faire une idée des attentes paysagères des différents groupes d'agents concernés par le paysage de la zone, il n'en demeure pas moins qu'ils ne permettent pas d'être intégrés dans les processus de décision publique qui s'appuient sur des indicateurs de bien-être agrégés. D'autant plus que, en se situant dans un contexte décisionnaire utilitariste, il est couramment admis que ces décisions publiques sont prises sur la base d'un calcul coût-bénéfice qui, au regard de ces premiers résultats, pourrait être fortement interrogé.

\section{Processus de décision publique et méthode des choix multi-attributs}

Certes, l'intérêt d'une évaluation économique est de pouvoir fournir des informations aux décideurs. Mais le contexte dans lequel prend ancrage leur processus de prise de décision justifie du type d'informations attendu. C'est pourquoi 
nous reviendrons tout d'abord sur ce contexte décisionnaire avant de s'attacher, selon le contexte retenu, à mettre en évidence les indicateurs nécessaires aux décideurs dans le cadre de ce processus de décision. Nous simulerons à titre illustratif différents programmes paysagers envisageables sur le site des Monts d'Arrée afin de pouvoir employer les indicateurs retenus comme support à la décision publique.

\section{Contexte décisionnaire}

La distinction communément faite en théorie politique oppose deux approches. Selon l'approche utilitariste, les décideurs choisissent parmi plusieurs alternatives celle qui maximise le bien-être social, celui-ci se définissant dans le cadre de la souveraineté du consommateur, c'est-à-dire à partir de la satisfaction des préférences individuelles de l'individu. Ainsi, pour la théorie du bien-être, "the purpose of economic activity is to increase the well-being of the individuals that make up the society and that each individual is the best judge of how well-off he or she is in given situation. " (Freeman III, 1993 , p. 3).

Si le processus de décision publique adopte l'approche déontologique (ou kantienne), c'est une logique de «mise en situation » qui prime, autrement dit les décideurs suivent les règles adaptées aux situations qu'ils rencontrent (March, 1994). Dans ce cas, ce n'est pas un individu-maximisateur (ou consommateur) qui fournit l'information aux politiques, mais un individu-citoyen qui ne se prononce que sur ce qu'il juge jusque-là bon pour la société.

Si l'instrument servant de support à la décision «utilitariste» est la méthode d'évaluation et les mesures de bénéfices auxquelles elle aboutit, il s'agit dans la vision kantienne du débat, de la négociation et du consensus concernant le bien commun. Nous poursuivrons donc la démarche générale de cette étude en envisageant un contexte décisionnaire utilitariste pour lequel les indicateurs souhaités sont calculables à partir du modèle développé dans la section précédente.

Dans ce contexte, l'analyse privilégiée pour une aide à la décision concernée par des biens environnementaux non-marchands est l'analyse coût-bénéfice (ou analyse coût-avantage (ACA)). Dans un cadre ex ante, et adapté au paysage, cette approche consiste à mettre en balance les coûts et les bénéfices qui seraient générés par la mise en œuvre d'une mesure paysagère particulière.

Si les décideurs peuvent être intéressés par la connaissance des préférences paysagères de catégories d'individus distinctes, les indicateurs qu'ils sont amenés à utiliser doivent être globaux et nécessitent donc d'être agrégés. La question de l'agrégation des préférences individuelles se pose donc pleinement: quel système de pondération des indicateurs individuels peut-on employer pour disposer d'un indicateur agrégé ?

\section{L'agrégation en question}

Le système de pondération que nous retenons sera explicité au travers du calcul du prix implicite agrégé par attribut paysager, ce qui nous permettra dans la section suivante de calculer le surplus compensateur, indicateur des bénéfices attendus de mesures paysagères et directement utilisable par le décideur. 
Le système d'agrégation retenu s'appuie sur une hypothèse forte de ce travail : le temps passé au contact du paysage influence la valeur qu'on lui accorde. Cette hypothèse, mise en parallèle avec les indicateurs monétaires calculés précédemment, revient à dire que la satisfaction retirée d'une mesure agissant sur le paysage dépend du temps que l'individu passe au contact de ce paysage, autrement dit dépend de sa connaissance du lieu (Dachary-Bernard, 2004).

Nous proposons donc d'agréger linéairement les CAP marginaux (ou prix implicites $P I$ ) en accordant un poids différent aux prix implicites des différentes populations enquêtées selon leur rapport au paysage. Rappelons à ce stade que la distinction résidents permanents/résidents secondaires opère pour l'attribut lande, ce qui nécessite de procéder à une agrégation particulière pour cet attribut. Le système d'agrégation retenu formalise donc le prix implicite agrégé du niveau $i$ de l'attribut $j\left(P I^{A}{ }_{j i}\right)$ de la façon suivante :

$$
\begin{aligned}
& P I_{j i}^{A}=\alpha P I_{j i}^{R}+\beta P I_{j i}^{T} \quad \forall i, \forall j \neq \text { lande } \\
& P I_{j i}^{A}=\alpha^{\prime} P I_{j i}^{R P}+\beta^{\prime} P I_{j i}^{R S}+\gamma^{\prime} P I_{j i}^{T} \quad \forall i, \forall j=\text { lande }
\end{aligned}
$$

où $P I^{R}{ }_{j i}$ est mis pour le prix implicite moyen des résidents, $P I^{T}{ }_{j i}$ pour celui des touristes, $P I^{R P}{ }_{j i}$ pour celui des résidents permanents et $P I^{R S}{ }_{j i}$ pour celui des résidents secondaires.

Ce sont les valeurs des paramètres $\alpha, \beta, \alpha^{\prime}, \beta^{\prime}$ et $\gamma^{\prime}$ que nous allons chercher à déterminer de manière à respecter les conditions suivantes :

$$
\left\{\begin{array}{l}
\alpha+\beta=1 \\
\alpha^{\prime}+\beta^{\prime}+\gamma^{\prime}=1
\end{array}\right.
$$

Nous supposons que la durée moyenne de séjour (touristes), de présence dans l'habitation secondaire (résident secondaire) ou de temps libre (habitant permanent) est entièrement passée au contact du paysage, même si le paysage n'est pas l'objet premier de l'activité de loisir pratiquée ${ }^{10}$. Compte tenu des caractéristiques des groupes d'individus, le tableau suivant indique la part du temps annuel supposé

\begin{tabular}{|c|c|c|c|c|c|}
\hline & & \multicolumn{2}{|c|}{ Nombre de jours par an } & \multicolumn{2}{|c|}{$\%$ du temps annuel } \\
\hline \multicolumn{2}{|c|}{ Touristes } & \multicolumn{2}{|c|}{7,5} & \multicolumn{2}{|c|}{$2 \%$} \\
\hline \multirow[t]{2}{*}{ Résidents } & secondaires & \multirow{2}{*}{193,8} & 96,7 & \multirow{2}{*}{$53,1 \%$} & $26,5 \%$ \\
\hline & & & 213,7 & & $58,5 \%$ \\
\hline
\end{tabular}
passé au contact du paysage des Monts d'Arrée.

Tableau 5. Temps passé au contact du paysage

10 Ces hypothèses sont certes très fortes, notamment concernant la population des résidents permanents, mais elles permettent d'envisager une pondération compte tenu des données disponibles caractérisant les populations étudiées. 
A partir de ces résultats, nous pouvons résoudre les deux systèmes suivants :

$$
\left\{\begin{array} { l } 
{ \alpha + \beta = 1 } \\
{ \beta = a \alpha }
\end{array} \quad \left\{\begin{array}{l}
\alpha^{\prime}+\beta^{\prime}+\gamma^{\prime}=1 \\
\beta^{\prime}=a^{\prime} \alpha^{\prime} \\
\gamma^{\prime}=b^{\prime} \alpha^{\prime}
\end{array}\right.\right.
$$

où $a, a^{\prime}$ et $b^{\prime}$ traduisent respectivement la durée moyenne de temps libre des touristes par rapport aux résidents, des secondaires par rapport aux permanents et des touristes par rapport aux permanents. On obtient :

$$
\Leftrightarrow\left\{\begin{array} { l } 
{ \alpha = 0 , 9 6 } \\
{ \beta = 0 , 0 4 }
\end{array} \Leftrightarrow \left\{\begin{array}{l}
\alpha^{\prime}=0,673 \\
\beta^{\prime}=0,303 \\
\gamma^{\prime}=0,024
\end{array}\right.\right.
$$

Les prix implicites agrégés sont donc calculés à partir des équations suivantes :

$$
\begin{aligned}
& P I_{j i}^{A}=0,96 P I_{j i}^{R}+0,04 P I_{j i}^{T} \quad \forall i, \forall j \neq \text { lande } \\
& P I_{j i}^{A}=0,673 P I_{j i}^{R P}+0,303 P I_{j i}^{R S}+0,024 P I_{j i}^{T} \quad \forall i, \forall j=\text { lande }
\end{aligned}
$$

Ce système de pondération accorde bien, comme il était souhaité au départ, plus de poids aux individus dont le temps passé au contact du paysage est plus élevé, privilégiant de ce fait les préférences paysagères des «connaisseurs » c'est-à-dire des agents susceptibles d'être plus sensibilisés aux transformations paysagères envisagées.

\begin{tabular}{|c|c|}
\hline Niveau d'attribut & PI agrégé $(€ /$ ménage/an) \\
\hline Lande $_{1}$ & $+17,7$ \\
\hline Lande $_{2}$ & $+19,1$ \\
\hline Lande $_{3}$ & $-37,9$ \\
\hline$\overline{\text { Bocage }_{1}}$ & $-36,3$ \\
\hline Bocage $_{2}$ & -7 \\
\hline Bocage $_{3}$ & $+43,5$ \\
\hline Bâti ${ }_{1}$ & +16 \\
\hline Bâti $i_{2}$ & -26 \\
\hline Bâti ${ }_{3}$ & +8 \\
\hline
\end{tabular}

Le tableau ci-dessous présente ces prix implicites agrégés pour chaque niveau d'attribut.

Tableau 6. Prix implicites agrégés par niveau d'attribut paysager

Ainsi, le ménage moyen confronté au paysage des Monts d'Arrée accepterait de payer $17,7 €$ par an pour que la lande soit dans son état très arboré. Le fait de ne pas entretenir la lande, ce qui, pour des décideurs, signifie un gain évident grâce aux coûts directs d'entretien évités, génère par ailleurs un bénéfice en terme de bien-être 
procuré aux usagers. A l'inverse, toute mesure visant à entretenir la lande pour qu'elle soit rase et non arborée génère, outre les coûts directs d'entretien, un coût supplémentaire du fait de la perte de bien-être que cette mesure occasionne chez les individus. Ce coût supplémentaire correspond à l'indemnité que les ménages souhaiteraient recevoir pour accepter un paysage de lande rase, soit environ $38 €$ par an et par ménage concerné.

Au niveau du bocage, les individus acceptent de payer annuellement 43,5€ par ménage pour que l'espace agricole soit fortement bocager; il s'agit du prix implicite le plus élevé. Ainsi, toute mesure politique cherchant à replanter des haies bocagères et/ ou entretenir des talus procurerait une amélioration du bien-être qui, additionnée à tout autre bénéfice associé à la mesure, pourrait être comparée à l'ensemble des coûts générés par la mesure. La pertinence économique d'une telle mesure serait ainsi estimée. En revanche, toute mesure «moyenne » sur le bocage serait associée à une perte de bien-être des individus évaluée à $7 €$ par an et par ménage : il s'agit du montant que les agents souhaiteraient se voir offrir en contrepartie de la mise en œuvre de la mesure.

Pour l'intégration du bâtiment agricole, en écartant de l'analyse le prix implicite associé au niveau 1 de l'attribut en raison de sa non-significativité chez les résidents, on peut estimer le bénéfice généré par toute mesure politique d'intégration totale des bâtis, et, de la même manière, la perte de bien-être associée à une intégration partielle. On peut également noter que le CAR associé à une intégration partielle des bâtiments est largement plus élevé en valeur absolue que le CAP d'une bonne intégration paysagère. A partir de ces indicateurs agrégés et connaissant les coûts de l'intégration partielle et complète, les décideurs pourront donc conclure sur la politique optimale d'intégration des bâtiments agricoles : compte tenu des coûts, la mise en œuvre d'une politique partielle pourrait s'avérer économiquement plus soutenable, malgré la perte de bien-être «social » qu'elle génèrerait, que la mesure totale.

Les prix implicites sont des taux marginaux de substitution entre un niveau d'attribut et une quantité d'argent. Ce ne sont cependant toujours pas les mesures de bien-être tant attendues par les décideurs pour choisir l'alternative politique permettant de dégager le bénéfice net maximal.

\section{Evaluation de bénéfices pour une analyse coûts-bénéfices}

En adoptant une mesure particulière, les décideurs cherchent à améliorer le bienêtre général, ce qui signifie que le surplus du consommateur doit être maximal. Or, les prix implicites calculés ne sont pas des estimateurs des surplus compensateurs nécessaires dans une analyse coût-bénéfice (Bennett and Blamey, 2001, p. 101).

Ce surplus, retiré de la mise en œuvre d'une alternative $N$, se définit de la façon suivante :

$$
C S_{N}=\frac{-1}{\partial V / \partial p}\left(V_{S Q}-V_{N}\right)
$$


où $V_{S Q}$ et $V_{N}$ sont respectivement mis pour l'utilité de la situation de référence (statu $q u o$ ) et de la nouvelle situation et le dénominateur correspond à l'utilité marginale de l'attribut monétaire sur le calcul de laquelle nous sommes revenus antérieurement: $-0,34$ pour les touristes et $-0,01$ pour les résidents. Les décideurs, en charge de la protection du paysage des Monts d'Arrée, vont donc pouvoir évaluer le gain ou la perte de bien-être générée par le passage de l'état initial (statu quo) à la nouvelle situation.

Cette situation du statu quo est caractérisée par les niveaux de base pour chacun des trois attributs: lande $_{1}$, bocage $_{1}$ et bâti ${ }_{1}$.

Le surplus initial du consommateur peut être calculé séparément pour les touristes et pour les résidents, compte tenu du fait que l'estimation de la fonction d'utilité indirecte a été réalisée pour chacune de ces deux classes de consommateurs. Le modèle retenu, comme présenté précédemment, est un Logit multinomial, ce qui implique que les caractéristiques individuelles sont intégrées dans le modèle. Les valeurs qu'il est conseillé de prendre pour ces dernières sont les valeurs moyennes $\overline{\nu p}=0,59$, enf $=1,5$ et $\overline{r v v}=1,48$.

Ainsi, les fonctions d'utilités indirectes des touristes $\left(V^{T}\right)$ et des résidents $\left(V^{R}\right)$ s'expriment de la façon suivante, en précisant que $\theta=1$ quand le scénario étudié est présenté en alternative 1 et que $\theta=0$ s'il l'est en option 2 :

$$
\begin{aligned}
& V^{T}=0,22 l_{2}-0,29 l_{3}+0,04 b_{2}+0,6 b_{3}-0,19 b_{a 2}+0,73 b_{a 3}+9,66 p-25 p^{2} \\
&+\theta * A_{1} *(-0,4 u p+0,24 e n f)+(1-\theta) * A_{2} *(-0,56 v p+0,26 e n f) \\
& V^{R}=+0,59 l_{2}-0,34 l_{3}-0,16 b_{2}+0,83 b_{3}-0,48 b_{a 2}+0,2 b_{a 3} \\
&+0,07 p-0,0009 p^{2}+\theta * A_{1} *\left(0,29 r v v+0,17 l_{2}-0,54 l_{3}\right) \\
&+(1-\theta) * A_{2} *\left(0,32 r v-0,37 l_{2}+0,24 r l_{3}\right)
\end{aligned}
$$

Le calcul du surplus retiré de la situation initiale (indicée $S Q$ selon l'équation (14)) considère plusieurs éléments dans le calcul : l'attribut monétaire $(\phi)$ est nul ; tous les attributs paysagers sont à leur niveaux de base (d'où la valeur -1 prise par toutes les variables représentant les niveaux 2 et 3 des attributs paysagers); aucune constante spécifique (ASC) ne joue au niveau du statu quo et par conséquent les caractéristiques individuelles (qui sont intégrées dans le modèle en interaction avec les ASC) ne jouent pas dans le calcul.

On trouve ainsi $V_{S Q}^{T}=-1,1$ et $V_{S Q}^{R}=-0,66$. Les bénéfices retirés par un touriste et un résident en présence de la situation de référence sont négatifs : il s'agit de perte de bien-être pour le cas où aucune intervention publique n'aurait lieu sur les Monts d'Arrée.

Le surplus associé à la situation initiale étant évalué, on peut désormais s'intéresser à l'estimation du surplus généré par une mesure paysagère particulière. 
Plusieurs programmes paysagers peuvent être étudiés, chacun d'eux étant défini à partir d'un niveau recherché pour chaque attribut paysager considéré. Dans le présent cas d'étude, il y aurait potentiellement six mesures «mono-attribut » (agissant sur un attribut uniquement), douze mesures «bi-attributs» (qui agissent sur deux attributs simultanément) et huit mesures «complètes » (qui s'intéressent aux trois attributs à la fois). Nous illustrerons la démarche proposée en nous focalisant sur quelques-unes de ces 26 mesures potentielles. Ce choix repose, d'une part, sur les actions paysagères envisagées par les décideurs locaux du PNRA et, d'autre part, sur les préférences paysagères des enquêtés révélées au travers des scénarii paysagers les plus fréquemment choisis.

Nous étudierons par conséquent les bénéfices retirés des cinq programmes $\mathrm{P}_{1}$ à $\mathrm{P}_{5}$ et répertoriés dans le tableau suivant.

Tableau 7. Programmes paysagers étudiés

\begin{tabular}{|c|c|c|}
\hline $\begin{array}{l}\text { Programmes } \\
\text { paysagers }\end{array}$ & $\begin{array}{c}\text { Niveau(x) } \\
\text { d'attributs visé(s) }\end{array}$ & Description \\
\hline $\mathrm{P}_{1}$ & lande $_{3}$ & Mesure de protection de la lande \\
\hline $\mathrm{P}_{2}$ & bocage $_{3}$, batti & Mesure de protection de l'espace agricole (en général) \\
\hline $\mathrm{P}_{3}$ & bocage, $_{2}$ batit ${ }_{3}$ & Mesure de protection de l'espace agricole (plus orientée vers les bâtis) \\
\hline $\mathrm{P}_{4}$ & $\begin{array}{l}\text { lande, }_{2} \text {, bocage } \\
\text { batti }\end{array}$ & Mesure « complète » orientée plus spécifiquement sur le bâti \\
\hline $\mathrm{P}_{5}$ & $\begin{array}{l}\text { landes, }_{3} \text { bocage } \\
\quad \text { batti }\end{array}$ & $\begin{array}{l}\text { Mesure « complète » orientée plus spécifiquement } \\
\text { sur les attributs naturels }\end{array}$ \\
\hline
\end{tabular}

D'après les équations (15) et (16), nous sommes en mesure de calculer les niveaux d'utilité indirecte pour chacun des programmes paysagers envisagés, puis d'en déduire les variations de surplus.

Le tableau 8 ci-après synthétise ces résultats (arrondis) par programme et selon le type de résident. Le tableau similaire concernant les touristes est reporté en annexe A3. A la lecture de ces résultats, nous pouvons faire une analyse en terme de bénéfice retiré par individu selon la mesure envisagée.

Tableau 8. Calcul des variations de bien-être pour les résidents

\begin{tabular}{lcccccc}
\hline & Statu quo & $\mathrm{P}_{1}$ & $\mathrm{P}_{2}$ & $\mathrm{P}_{3}$ & $\mathrm{P}_{4}$ & $\mathrm{P}_{5}$ \\
\hline Utilité indirecte des résidents permanents & $-0,64$ & 0,007 & 2,23 & 1,68 & 2,06 & 0,75 \\
\hline $\begin{array}{l}\text { Variation du surplus des résidents } \\
\text { permanent* }\end{array}$ & $\mathbf{x}$ & +323 & +1435 & +1162 & +1351 & +693 \\
\hline \begin{tabular}{l} 
Utilité indirecte des résidents secondaires \\
\hline $\begin{array}{l}\text { Variation du surplus des résidents } \\
\text { secondaires* }\end{array}$
\end{tabular} & $-0,64$ & 0,55 & 2,06 & 1,51 & 2,39 & 1,29 \\
\hline
\end{tabular}


Nous restreindrons l'analyse comparative aux deux programmes paysagers qui génèrent les plus fortes variations de surplus, à savoir les programmes $\mathrm{P}_{2}$ et $\mathrm{P}_{4}$.

La mesure de conservation de l'espace agricole cultivé $P_{2}$ entraîne une variation de bien-être positive pour les trois catégories d'agents. Les touristes ont ainsi un consentement à payer pour ce scénario de 9,63€ par an, consentement le plus élevé de tous les consentements à payer estimés les concernant. Ce programme de conservation est donc celui qui génèrerait le bénéfice le plus important auprès des touristes. C'est également le cas des habitants permanents, dont le consentement à payer pour $\mathrm{P}_{2}$ s'élève à $1435 €$ par an. Les secondaires dégagent un fort CAP annuel, de $1348 €$, mais cela reste encore inférieur à celui qu'ils attribuent à un autre programme d'actions paysagères. Globalement, l'état de l'espace agricole est très fortement valorisé par les enquêtés, qui souhaitent un espace très bocager et des bâtiments intégrés dans le paysage.

Le programme $\mathrm{P}_{4}$ agit prioritairement sur le bâti (qu'il intègre parfaitement), plus faiblement sur les deux autres attributs. Il est véritablement «multi-attributs » dans le sens où il agit sur les trois attributs simultanément. Les touristes retireraient de cette mesure un gain tel qu'ils consentent à payer 8,2€ par an. Les résidents permanents ont un consentement à payer annuel pour ce programme d'actions paysagères de $1351 €$, et les habitants secondaires de $1513 €$. Il s'agit, pour ces derniers, du programme le plus avantageux, en raison, notamment, de leur forte préférence pour une lande moyennement arborée.

Les pouvoirs publics en charge de prendre la décision du choix de la mesure «optimale» d'un point de vue coût-bénéfice doivent comparer ces différents bénéfices avec les coûts de mise en œuvre de ces mesures. Il est donc nécessaire que les décideurs disposent d'un bénéfice agrégé.

Le système de pondération retenu pour agréger les bénéfices est le même que celui précédemment employé pour le calcul des prix implicites agrégés $\left(\alpha^{\prime}, \beta^{\prime}, \gamma^{\prime}\right)$. Le calcul du bénéfice agrégé doit également prendre en compte le nombre d'individus concernés puisque les surplus calculés précédemment sont des surplus individuels, pour le ménage moyen de la catégorie d'agents concernée. Ainsi, le surplus agrégé de la mise en place de la mesure $N$ est le suivant :

$$
C S_{N}=\left(\alpha^{\prime} \cdot C S_{N}^{R P} \cdot R P\right)+\left(\beta^{\prime} \cdot C S_{N}^{R S} \cdot R S\right)+\left(\gamma^{\prime} \cdot C S_{N}^{T} \cdot T\right)
$$

$R P, R S$, et $T$ représentent respectivement le nombre de ménages permanents, de ménages secondaires et de ménages touristes.

D’après les données du recensement général de la population (1999) produites par l'INSEE, on évalue, sur la zone d'étude, le nombre de résidences permanentes à 4911 et le nombre de résidences secondaires à $1579^{11}$. Le logement (permanent ou secondaire) étant associé à un ménage, ces chiffres seront ceux du nombre de ménages respectivement permanents et secondaires. Par ailleurs, le nombre de ménages touristes

${ }^{11}$ Il s'agit du nombre de résidences secondaires et de logements occasionnels. 
est difficile à évaluer puisque nous ne disposons pas de chiffres officiels. Par conséquent, nous simulerons plusieurs surplus agrégés en fonction de plusieurs niveaux de $T$, nombre de ménages touristes concernés par le supplément de taxe proposé (c'est-à-dire hors itinérants). Nous prendrons successivement $T_{1}=25000$; $T_{2}=50000 ; T_{3}=75000$.

Sachant d'autre part que $\left(\alpha^{\prime}, \beta^{\prime}, \gamma^{\prime}\right)=(0,673 ; 0,303 ; 0,024)$, nous sommes en mesure d'estimer l'équation (17) dont les résultats sont fournis dans le tableau 9 suivant $\left(C S^{(t)}{ }_{t=1,2,3}\right.$ fait référence à la taille de la population de touristes $T_{t}$ considérée).

Tableau 9. Calculs des bénéfices agrégés par programme paysager $(\mathrm{en} €)$

\begin{tabular}{cccccc}
\hline & $\mathrm{P}_{1}$ & $\mathrm{P}_{2}$ & $\mathrm{P}_{3}$ & $\mathrm{P}_{4}$ & $\mathrm{P}_{5}$ \\
\hline $\mathrm{CS}^{(1)}$ & +1353309 & +5395529 & +4359168 & +5192691 & +2756024 \\
\hline $\mathrm{CS}^{(2)}$ & +1354033 & +5401318 & +4364338 & +5197632 & +2759554 \\
\hline$\Delta \mathrm{CS}_{(2 / 1)}$ & +724 & +5788 & +5171 & +4941 & +3529 \\
\hline $\mathrm{CS}^{(3)}$ & +1354756 & +5407106 & +4369509 & +5202573 & +2763084 \\
\hline$\Delta \mathrm{CS}_{(3 / 1)}$ & +1447 & +11576 & +10341 & +9882 & +7058 \\
\hline$\Delta \mathrm{CS}_{(3 / 2)}$ & +724 & +5788 & +5171 & +4941 & +3529 \\
\hline
\end{tabular}

Les trois simulations successives permettent de relativiser l'importance de la taille de la population de touristes. En effet, l'écart de surplus selon le nombre de ménages touristes considérés dans les calculs est relativement faible par rapport au montant total du bénéfice $(0,05 \%)$. La comparaison de ces surplus agrégés permet de constater que le programme $\mathrm{P}_{2}$, pour lequel les gains générés sont les plus importants, permettrait de dégager un bénéfice de plus de $5 \mathrm{M} €$. C'est donc ce montant que, d'après l'analyse coût-bénéfice, les décideurs devraient comparer aux coûts de la mise en œuvre de la politique $\mathrm{P}_{2}$ pour décider de l'intérêt économique de sa mise en ouvre.

\section{Conclusion}

Cet article avait une double prétention. La première était de présenter une méthode d'évaluation encore peu utilisée en France et particulièrement bien adaptée au paysage dans sa dimension multi-attributs. Le second objectif concernait l'intérêt d'une telle méthode au regard du processus d'aide à la décision publique dans lequel elle s'inscrit.

Concernant la première finalité, la réflexion a permis de montrer les avantages de la méthode des choix multi-attributs pour évaluer le paysage, ce dernier étant défini comme un bien économique non marchand, multi-attributs en ce sens qu'il procure du bien-être aux usagers de par ses différentes composantes. La MCMA est de ce fait adaptée puisqu'elle adopte elle-même une approche multi-attributs. Mais au-delà 
de cet aspect, l'emploi de la MCMA permet à l'évaluateur de disposer d'un éventail de préférences révélées. Un modèle de préférences paysagères a pu être développé pour les deux groupes d'individus enquêtés. L'usage de la MCMA a permis à ce stade de disposer de paramètres pour chaque niveau d'attributs considérés. De ce fait, les prix implicites pour chaque niveau d'attributs paysagers ont été calculés révélant, entre autre, une préférence partagée entre les agents pour une lande arborée. Ce résultat est intéressant au regard des mesures paysagères existantes sur la zone d'étude, puisque celles-ci s'orientent en effet vers une protection de la lande dans son état ras. Mais notons les perspectives d'enrichissement du modèle économétrique en proposant un mixed logit, qui permettrait, notamment, de s'intéresser plus en détails à l'hétérogénéité des enquêtés.

Concernant notre seconde ambition, l'article, après avoir repositionné la question de l'évaluation dans le cadre du processus de décision publique, a montré en quoi la MCMA apportait des outils intéressants aux décideurs. Ces derniers ont en effet besoin d'information par attribut, afin d'évaluer des mesures paysagères qui n'agiraient pas sur tous les attributs du paysage. Mais ils ont besoin d'une information agrégée par rapport aux préférences individuelles qui se sont dégagées de la modélisation. Notre démarche est donc revenue sur la question de l'agrégation des bénéfices individuels et, partant d'une hypothèse concernant les pondérations à donner, plusieurs programmes paysagers différents ont été étudiés. Ces résultats en terme de variation de surplus soulignent empiriquement le rôle de l'évaluation ex ante. Mais il est important de noter que nous ne disposons pas de l'information concernant les coûts de mise en œuvre des différents programmes. Il est donc à ce stade impossible de conclure sur ce que les décideurs devraient adopter comme mesure paysagère sur la zone. A la seule lecture des bénéfices agrégés, nous pouvons être tentés de diriger notre choix vers le programme générant les plus forts bénéfices. Or, il est très probable qu'un tel programme soit l'un des plus coûteux, voire le plus coûteux, et qu'au final, il ne dégage pas de bénéfice net. Il s'agit en effet d'une mesure visant à replanter les bocages et intégrer les bâtiments agricoles.

La réflexion menée dans cet article a contribué à alimenter le débat sur l'évaluation économique et tout particulièrement sur les biens environnementaux non marchands. L'évaluation doit être considérée comme un outil d'aide à la décision. C'est pourquoi, si cela implique la traduction en langage monétaire des valeurs accordées au paysage, cela ne signifie en rien que le paysage ait un "prix » ou qu'il soit souhaité de le «marchandiser». L'emploi de la MCMA est sur ce point particulièrement évocateur. Comme nous l'avons vu, sa logique multi-attributs conduit à fournir plusieurs indicateurs selon les transformations potentielles que connaitrait le paysage, autrement dit plusieurs «prix ». Si plusieurs «prix» sont associés au paysage, c'est bien la preuve qu'il ne fait pas l'objet d'une unique valorisation monétaire : une vérité de La Palisse en quelque sorte. 


\section{Bibliographie}

Adamowicz W., Louviere J. and Swait J. (1998). Introduction to attribute-based stated choice methods, Report for NOAA Resource valuation Brach, Edmonton (Canada), Damage Assessment Center.

Adamowicz W., Louviere J. and Williams M. (1994). Combining revealed and stated preference methods for valuing environmental amenities, Journal of Environmental Economics and Management, 26, pp. 271-292.

Aldred J. (2006). Incommensurability and monetary valuation, Land Economics, 82, pp. 141-161.

Alvarez-Farizo B., Hanley N. (2002). Using conjoint analysis to quantify public preferences over the environmental impacts of wind farms. An example from Spain, Energy Policy, 30 (2), pp. 107-116.

Bateman I., Carson R.T., Day B., Hanemann M., Hanley N., Tannis H., Jones-Lee M., Loomes G., Mourato S., Özdemiroglu E., Pearce D.W., Sugden R. and Swanson J. (2002). Economic Valuation with Stated Preference Techniques: A Manual, Cheltenham (UK) \& Northampton, Massachusetts (USA), Edward Elgar, in association with the UK Department for Transport, $458 \mathrm{p}$.

Bech M., Gyrd-Hansen D. (2005). Effects coding in discrete choice experiments, Health Economics, 14 (10), pp. 1079-1083.

Bennett J., Blamey R. (2001). The Choice Modelling Approach to Environmental Valuation, Cheltenham (UK), Edward Edgar Publishing Inc., 261 p.

Bergmann A., Hanley N. and Wright R. (2006). Valuing the attributes of renewable energy investments, Energy Policy, 34 (9), pp. 1004-1014.

Bonnieux F., Carpentier A. et Paoli J.-C. (2006). Priorités des résidents et des visiteurs pour la protection et l'aménagement de la forêt en Corse : exemple de la forêt de Bonifatu, Revue forestière française, 58 (2), pp. 167-180.

Boxall P., Adamowicz W., Swait J., Williams M. and Louviere J. (1996). A comparison of stated preference methods for environmental valuation, Ecological Economics, 18, pp. 243-253.

Bullock C.H., Kay J. (1997). Preservation and change in the upland landscape: The public benefits of grazing management, Journal of Environmental Planning and Management, 40, pp. 321-333.

Carlsson F., Frykblom P. and Liljenstolpe C. (2003). Valuing wetland attributes : An application of choice experiments, Ecological Economics, 47 (1), pp. 95-103.

Colombo S., Calatrava-Requena J. and Hanley N. (2006). Analysing the social benefits of soil conservation measures using stated preference methods, Ecological Economics, 58 (4), pp. 850-861. 
Conseil de l'Europe (2000). La Convention européenne du Paysage, Strasbourg, Conseil de l'Europe, 19 p.

Dachary-Bernard J. (2005). Une évaluation économique du paysage. Une application de la méthode des choix multi-attributs aux Monts d'Arée, Economie et Statistique, 373, pp. 57-74.

Dachary-Bernard J. (2004). Approche multi-attributs pour une évaluation économique du paysage, Thèse de Sciences économiques et sociales, Université MontesquieuBordeaux IV, sous la direction de P. Point, 290 p.

Foster V., Mourato S. (2003). Elicitation format and sensitivity to scope, Environmental and Resource Economics, 24, pp. 141-160.

Freeman III A.M. (1993). The Measurement of Environmental and Resource Values. Theory and Methods, Washington, DC, Resources for the Future, 516 p.

Godard O. (2004). Autour des conflits à dimension environnementale. Evaluation économique et coordination dans un monde complexe, Ecole PolytechniqueCNRS, Laboratoire d'économétrie, Paris, Cabier 2004-012, 23 p.

Gourmelen L. (2002). Construction et évolution des bocages des Monts d'Arrée, Cabiers scientifiques-Parc naturel régional d'armorique, 3, $7 \mathrm{p}$.

Graves P.E. (1991). Aesthetics, in: Measuring the Demand for Environmental Quality, Braden J. B., Kolstad C. D. (eds), Amsterdam, Elsevier, pp. 213-226.

Greene W.H. (2003). Econometric Analysis, Upper Saddle River, New Jersey (USA), Prentice-Hall International Inc., 1026 p.

Hanley N., MacMillan D., Wright R.E., Bullock C., Simpson I., Parsisson D. and Crabtree B. (1998a). Contingent valuation versus choice experiments: Estimating the benefits of environmentally sensitive areas in Scotland, Journal of Agricultural Economics, 49 (1), pp. 1-15.

Hanley N., Wright R.E. and Adamowicz W. (1998b). Using choice experiments to value the environment. Design issues, current experience and future prospects, Environmental and Resource Economics, 11 (3-4), pp. 413-428.

Hanley N., Wright R.E. and Koop G. (2000). Modelling recreation demand using choice experiments : Climbing in Scotland, Economics department, University of Glasgow, Discussion paper, $28 \mathrm{p}$.

Hensher D.A., Rose J.M. and Greene W.H. (2005). Applied Choice Analysis. A Primer, Cambridge, Cambridge University Press, 717 p.

Horne P., Boxall P.C. and Adamowicz W.L. (2005). Multiple-use management of forest recreation sites : A spatially explicit choice experiment, Forest Ecology and Management Decision Support in Multi-Purpose Forestry, 207 (1-2), pp. 189-199.

Krinsky I., Robb L.A. (1981). On approximating in statistical properties of elasticities, Review of Economics and Statistics, 68, pp. 715-719. 
Kuhfeld W.F. (2000). Multinomial Logit, discrete choice modelling - An introduction to designing choice experiments, and collecting, processing and analysing choice data with the SAS system, SAS Institute Inc. (www.sas.com), $308 \mathrm{p}$.

Lancaster K.J. (1971). Consumer Demand: A New Approach, New York, Columbia University Press, $177 \mathrm{p}$.

Le Floch S. (2000). Multifor program : final report on the qualitative survey. French case-study, area 1, Monts d'Arrée (Brittany region), Cemagref, Bordeaux, rapport de programme européen, $66 \mathrm{p}$.

Louviere J., Hensher D.A. and Swait J.D. (2000). Stated Choice Methods. Analysis and Application, Cambridge (UK), Cambridge University Press, 402 p.

Louviere J., Woodworth G. (1983). Design and analysis of simulated consumer choice or allocation experiments: An approach based on aggregate data, Journal of Marketing Research, 20, pp. 350-367.

March J.G. (1994). A Primer in Decision Making, New York, The Free Press, 289 p.

Mcfadden D. (1974). Conditional logit analysis of qualitative choice behaviour, in: Frontiers in Econometrics, P. Zarembka. (ed.), New York, Academic Press, pp. 105-142.

McVittie A., Hanley N. and Oglethorpe D. (2001). Choice experiments, benefits transfert and the design of agri-environmental policy, Agricultural Economics Society one day conference: "Choice experiments: A new approach to environmental valuation », 9 April, London, $21 \mathrm{p}$.

Morrison M., Bennett J. and Blamey R.K. (1999). Valuing improved wetland quality using choice modeling, Water Resources Research, 35 (9), pp. 2805-2814.

Parcs naturels régionaux de France (2000). Paysages de bocage, Gestion des espaces naturels, agricoles et forestiers, Fédération des parcs naturels régionaux de France, Collection Expérimenter pour agir, $42 \mathrm{p}$.

Poe G.L., Welsh M. and Champ P. (1997). Measuring the difference in mean willingness to pay when dichotomous choice contingent valuation responses are not independant, Land Economics, 73 (2), pp. 255-267.

Provencher B., Bishop R.C. (2004). Does accounting for preference heterogeneity improve the forecasting of a random utility mode? A case study, Journal of Environmental Economics and Management, 48, pp. 793-810.

Rigby D., Burton M. (2004). Modeling indifference and dislike : a bounded bayesian mixed logit model of the UK market for GM food, Agricultural Economics Society $78^{\text {th }}$ Annual Conference, 2-4 april, London, 29 p.

Thurstone L. (1927). A law of comparative judgement, Psychological Review, 4, pp. 273-286. 
Train K. (2002). Discrete Choice Methods with Simulation, Cambridge (UK), Cambridge University Press.

Willis K.G., Garrod G.D. (1993). Valuing landscape: A contingent valuation approach, Journal of Environmental Management, 37, pp. 1-22.

Wilson E.O. (1998). Consilience: The Unity of Knowledge, New York, Alfred Knopf, $384 \mathrm{p}$. 


\section{ANNEXES \\ A0. Question posée aux résidents lors de la présentation des expériences de choix}

On vous demande pour les questions qui suivent de vous «mettre en situation », puisque tout ce que nous allons vous présenter est hypothétique.

A chaque scénario correspond une qualité paysagère qui prévaudrait si l'on met en place aujourd'hui une politique active en matière de paysage.

Trois éléments paysagers sont en jeu : le bocage, la lande et le bâti agricole (non traditionnel).

Chaque scénario proposé a un coût que votre ménage serait appelé à financer en partie par le biais d'un supplément de taxe d'habitation. Aujourd'hui, la taxe moyenne d'habitation sur les Monts d'Arrée est de $450 €$ (ou 3000 francs) par an et par ménage. La politique du paysage proposée supposerait une augmentation de cette taxe pour un montant de $15 €, 30 €$ ou $45 €$ par an et par ménage.

Le scénario numéroté 3 (ou statu quo) correspond à la situation dans laquelle aucune action paysagère n'est entreprise (et donc aucun coût).

Dans ce contexte, quel est le scénario paysager que vous préférez, si vous en préférez un, pour chacun de ces 6 ensembles?

\section{A1. Exemple d'expérience (ensemble 1) présentée aux résidents : 2 scénarii alternatifs et le statu quo (non-intervention)}

Ensemble 1 (scénario 1)

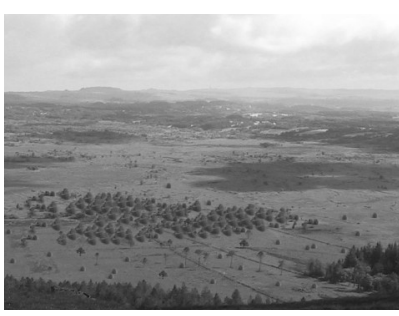

Lande peu arborée

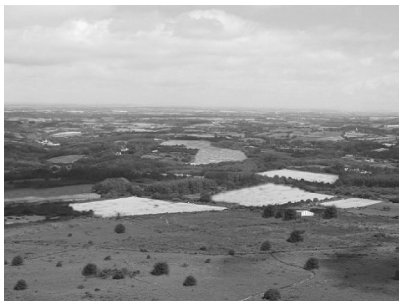

Espace agricole peu bocager Bâtiments agricoles peu intégrés
Ensemble 1 (scénario 2)

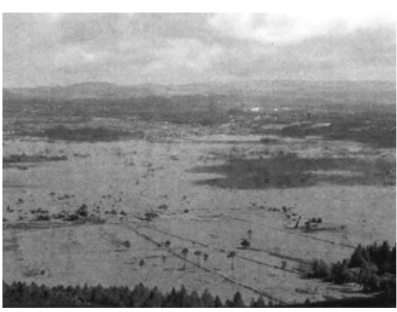

Lande rase

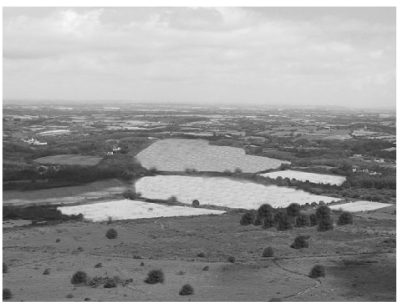

Espace agricole non bocager

Bâtiments agricoles bien intégrés
Statu quo

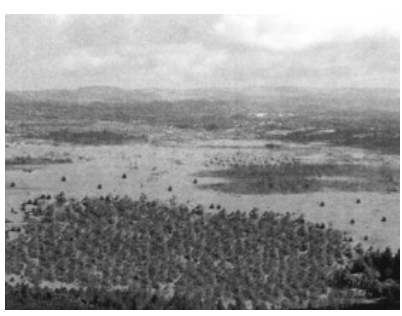

Lande très arborée

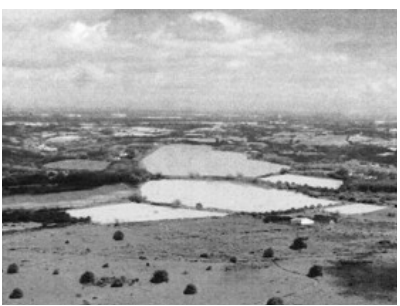

Espace agricole non bocager

Bâtiments agricoles non intégrés 


\section{A2. Descriptif des caractéristiques individuelles envisagées dans la modélisation initiale}

\begin{tabular}{|c|c|c|c|}
\hline $\begin{array}{l}\text { Population } \\
\text { concernée }\end{array}$ & $\begin{array}{c}\text { Libellé } \\
\text { de la variable } \\
\text { socioéconomique }\end{array}$ & Signification de la variable & Modalités \\
\hline Touristes / Résidents & sexe & Sexe de l'individu & $\begin{array}{l}0: \text { homme } \\
1: \text { femme }\end{array}$ \\
\hline Touristes / Résidents & âge & Âge de l'individu & en années \\
\hline Touristes / Résidents & nombre d'enfants & Nombre d'enfants dans le ménage & $0,1,2,3$ ou 4 (pour 4 enfants ou plus) \\
\hline Touristes / Résidents & revenu & $\begin{array}{l}\text { Tranche de revenu mensuel } \\
\text { du ménage en euros }\end{array}$ & $\begin{array}{l}0:<1000 € \\
1:[1000 €-1500 €] \\
2:[1500 €-3000 €] \\
3:[3000 €-4000 €] \\
4:>4000 €\end{array}$ \\
\hline Touristes / Résidents & cadrehab & $\begin{array}{l}\text { Cadre de l'habitation principale } \\
\text { de l'individu }\end{array}$ & $\begin{array}{l}0: \text { rural } \\
1: \text { urbain }\end{array}$ \\
\hline Touristes & muitées & Nombre de nuits passées sur la zone & $\begin{array}{l}0: \text { aucune } \\
1: \text { une nuit } \\
2: \text { moins d'une semaine } \\
3: \text { entre une et deux semaines } \\
4: \text { plus de deux semaines }\end{array}$ \\
\hline Touristes & visitepa & $\begin{array}{l}\text { Variable indiquant si c'est la première } \\
\text { visite sur la zone }\end{array}$ & $\begin{array}{l}0: \text { individu déjà venu } \\
1: \text { c'est la première visite }\end{array}$ \\
\hline Résidents & zone & $\begin{array}{l}\text { Zone d'implantation du logement } \\
\text { de l'enquêté sur le terrain d'étude }\end{array}$ & $\begin{array}{l}0: \text { Nord } \\
1: \text { Sud }\end{array}$ \\
\hline Résidents & Res_prin & Qualité de résidence de l'individu & $\begin{array}{l}0: \text { résident secondaire } \\
1: \text { résident principal }\end{array}$ \\
\hline Résidents & $M A$ & $\begin{array}{l}\text { Origine des Monts d'Arrée pour } \\
\text { l'individu (naissance) }\end{array}$ & $\begin{array}{l}0: \text { non originaire des Monts d'Arrée } \\
1: \text { originaire des Monts d'Arrée }\end{array}$ \\
\hline Résidents & ancienne & $\begin{array}{l}\text { Variable indiquant l'ancienneté } \\
\text { d'occupation du lieu par l'individu }\end{array}$ & $\begin{array}{l}0: \text { première année } \\
1: \text { entre } 2 \text { et } 5 \text { ans } \\
2: \text { plus de } 5 \text { ans }\end{array}$ \\
\hline
\end{tabular}




\section{A3. Evaluation des préférences paysagères des touristes et évaluation de mesures politiques paysagères. Synthèse des résultats (Dachary-Bernard, 2005)}

\begin{tabular}{|c|c|c|c|c|c|}
\hline Variables & & $\begin{array}{l}\text { Paramètres } \\
\text { estimés }\end{array}$ & $\begin{array}{l}\text { Paramètres } \\
\text { calculés }\end{array}$ & $\begin{array}{c}P I \\
\text { (€/personne/nuit) }\end{array}$ & $\begin{array}{c}P I \\
\text { (€/ménage/an) }\end{array}$ \\
\hline \multicolumn{6}{|l|}{ Attributs: } \\
\hline Lande $_{1}$ & $\left(\beta_{0}\right)$ & & $+0,07 /$ & $\begin{array}{c}-0,007 \\
(-0,28 ; 0,26)\end{array}$ & $-0,2$ \\
\hline Lande $_{2}$ & $\left(\beta_{1}\right)$ & $+0,22 * *$ & & $\begin{array}{c}+0,09 \\
(-0,67 ; 0,73)\end{array}$ & $+2,4$ \\
\hline Lande $_{3}$ & $\left(\beta_{2}\right)$ & $-0,29 * * *$ & & $\begin{array}{c}-0,16 \\
(-1 ; 1,05)\end{array}$ & $-4,2$ \\
\hline Bocage $_{1}$ & $\left(\delta_{0}\right)$ & & $-0,64 * *$ & $\begin{array}{c}-0,31 \\
(-2,1 ; 2,01)\end{array}$ & $-8,1$ \\
\hline Bocage $_{2}$ & $\left(\delta_{1}\right)$ & $+0,04 /$ & & $\begin{array}{c}+0,06 \\
(-0,06 ; 0,2)\end{array}$ & $+1,6$ \\
\hline Bocage $_{3}$ & $\left(\delta_{2}\right)$ & $+0,6 * * *$ & & $\begin{array}{c}+0,25 \\
(-2,04 ; 2)\end{array}$ & $+6,6$ \\
\hline Bâti-agricole $_{1}$ & $\left(\eta_{0}\right)$ & & $-0,54 * *$ & $\begin{array}{c}-0,22 \\
(-1,52 ; 1,49)\end{array}$ & $-5,8$ \\
\hline Bâti-agricole 2 & $\left(\eta_{1}\right)$ & $-0,19 /$ & & $\begin{array}{c}-0,07 \\
(-0,67 ; 0,55)\end{array}$ & $-1,8$ \\
\hline Bâti-agricole 3 & $\left(\eta_{2}\right)$ & $+0,73 * * *$ & & $\begin{array}{c}+0,38 \\
(-2,1 ; 2,25)\end{array}$ & +10 \\
\hline Prix & $\left(\pi_{1}\right)$ & $+9,66 * *$ & & & \\
\hline $\operatorname{Prix}^{2}$ & $\left(\pi_{2}\right)$ & $-25 * *$ & & & \\
\hline \multicolumn{6}{|l|}{ Caractéristiques: } \\
\hline Visitepassée $\left(\mathrm{ACS}_{1}\right)$ & $\left(\gamma_{11}\right)$ & $-0,4 /$ & & & \\
\hline Visitepassée $\left(\mathrm{ACS}_{2}\right)$ & $\left(\gamma_{12}\right)$ & $-0,56 * *$ & & & \\
\hline Nbreenf( $\left(\mathrm{ACS}_{1}\right)$ & $\left(\gamma_{31}\right)$ & $+0,24 * * *$ & & & \\
\hline Nbreenf $\left(\mathrm{ACS}_{2}\right)$ & $\left(\gamma_{32}\right)$ & $+0,26 * * *$ & & & \\
\hline
\end{tabular}

Significativité à $1 \%(* * *), 5 \%(* *), 10 \%(*)$ ou non-significativité $(/)$ $95 \%$ intervalle de confiance des prix implicites d'après Poe et al., 1997

\begin{tabular}{lcccccc}
\hline & Statu quo & $\mathrm{P}_{1}$ & $\mathrm{P}_{2}$ & $\mathrm{P}_{3}$ & $\mathrm{P}_{4}$ & $\mathrm{P}_{5}$ \\
\hline Utilité indirecte des touristes & $-1,1$ & $-0,69$ & 2,18 & 1,83 & 1,70 & 0,90 \\
\hline Variation du surplus des touristes & $\times$ & $+1,2$ & $+9,6$ & $+8,6$ & $+8,2$ & $+5,9$ \\
\hline
\end{tabular}

